Analytical queries on semantic trajectories using graph databases Peer-reviewed author version

Gomez, Leticia, I; KUIJPERS, Bart \& VAISMAN, Alejandro (2019) Analytical queries on semantic trajectories using graph databases. In: TRANSACTIONS IN GIS,.

DOI: $10.1111 /$ tgis. 12556

Handle: http://hdl.handle.net/1942/29137 


\title{
Analytical Queries on Semantic Trajectories using Graph Databases
}

\author{
Leticia Gómez ${ }^{1}$, Bart Kuijpers², Alejandro Vaisman ${ }^{3}$
}

\begin{abstract}
This paper studies the analysis of moving object data collected by location-aware devices, such as GPS, using graph databases. Such raw trajectories can be transformed into so-called semantic trajectories, which are sequences of stops that occur at "places of interest." Trajectory data analysis can be enriched if spatial and non-spatial contextual data associated with the moving objects are taken into account and aggregation of trajectory data can reveal hidden patterns within such data.

When trajectory data are stored in relational databases, there is an "impedance mismatch" between the representation and storage models. Graphs in which the nodes and edges are annotated with properties, are gaining increasing interest to model a variety of networks. Therefore, this paper proposes the use of graph databases (Neo4j in this case) to represent and store trajectory data, which can thus be analysed at different aggregation levels using graph query languages (Cypher, for Neo4j). Through a real-world public data case study, the paper shows that trajectory queries are expressed more naturally on the graph-based representation than over the relational alternative, and perform better in many typical cases.
\end{abstract}

Keywords: Trajectories, Semantic Trajectories, OLAP, Graph Databases, Graph OLAP, Graph Aggregation, Trajectory Analysis

\section{Introduction and Motivation}

Moving object data (MOD) applications Güting and Schneider2005 are a relevant topic for the GIS (Geographic Information Systems) community since a long time. The behaviour of moving objects can be traced

\footnotetext{
${ }^{1}$ Instituto Tecnológico de Buenos Aires, Buenos Aires, Argentina; email: lgomez@itba.edu.ar

${ }^{2}$ Hasselt University, Belgium; email: bart.kuijpers@uhasselt.edu

${ }^{3}$ Instituto Tecnológico de Buenos Aires, Buenos Aires, Argentina; email: avaisman@itba.edu.ar
} 
using location-aware devices (e.g., GPS, RFID). This produces trajectory data, which can be analysed in order to obtain interesting mobility patterns Renso et al.2013. The trajectory of a moving object is given by samples consisting of a finite number of $\langle O i d, t, x, y\rangle$-tuples, meaning that at a moment in time $t$, the object with identifier Oid is located at coordinates $(x, y)$. Over these data, different kinds of analyses can be performed Giannotti et al.2006, Giannotti et al.2007, Karli and Saygin2009. Trajectory analysis can not only be performed over the original (raw) trajectories, but also over a database built based on the ideas introduced by Spaccapietra et al. [Spaccapietra et al.2008, Parent et al.2013, where it is assumed that objects move over a background map consisting of disjoint geometrical figures to which semantically meaningful attributes are associated. These geometrical figures are referred to as Places of Interest (PoIs) of the application. Typically, they depend on the application area. In a tourist application, usual examples of PoIs are restaurants, historical buildings and hotels, while for traffic analysis PoIs could be defined as interesting road junctions or large parking lots. A PoI is considered a stop, when a moving object remains in it for a duration above some threshold, in which case all $(x, y)$-points of a trajectory that are located inside the PoI are transformed to the spatial object that represents this stop. As such, each object's trajectory, being a sequence of points, can be transformed into a sequence of stops. Thus, trajectory analysis can be applied to these transformed trajectories, which are called semantic trajectories, given that they can provide more information than the one provided by the $(t, x, y)$-points alone.

The intuition of the problem addressed in this paper is given next. Figure 1 (left) shows a simplified version of part of the map of London, which shows two hotels, indicated as Hotel 1 and Hotel 2 in the figure and abbreviated to $\mathrm{H} 1$ and $\mathrm{H} 2$ in the tables. The map also shows St Paul's Cathedral and the Buckingham Palace. Further, the map also shows the movement of the objects O1, O2 and O3. Object O1 moves from Hotel 1 to the Cathedral, then to the Palace, where its remains for some time, and then returns to its hotel. Object $\mathrm{O} 2$ moves from Hotel 2 to the Cathedral, next to the Palace (where it spends a few hours) and finally returns to its hotel. Object O3 leaves Hotel 2, visits the Palace, and returns to Hotel 2. Figure 1 (center) shows a portion of a table containing the raw trajectories (i.e., expressed for each object as $\langle t, x, y\rangle$-tuples). Figure 1 (right) gives the table with the PoIs corresponding to the application (details of how these tables are obtained are beyond the scope of the paper). The points belonging to the same trajectory are temporally ordered, and identified by an object identifier. In this setting, a data scientist may pose queries like "How many persons went from H1 to St Paul's Cathedral, and then to the Buckingham Palace (stopping to visit both places) during the same day." An analyst may also want to identify 


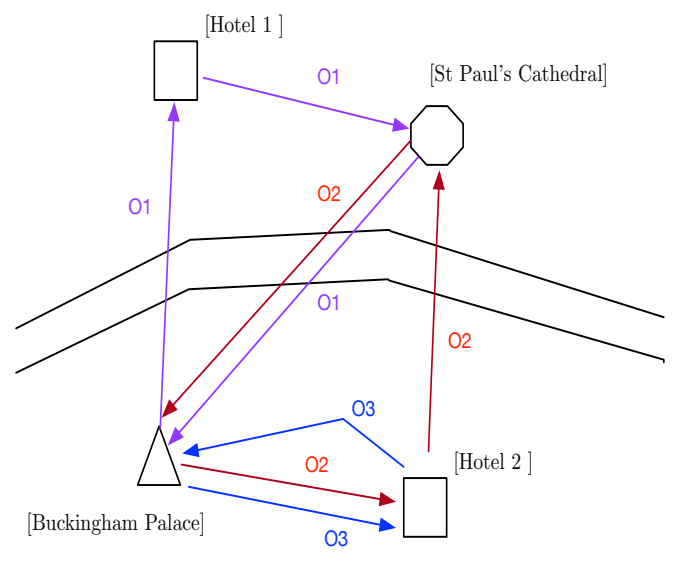

\begin{tabular}{|c|c|c|c|}
\hline$O_{i d}$ & $t$ & $x$ & $y$ \\
\hline$O_{1}$ & 1 & $x_{1}$ & $y_{1}$ \\
$O_{1}$ & 2 & $x_{2}$ & $y_{2}$ \\
$O_{1}$ & 3 & $x_{3}$ & $y_{3}$ \\
$O_{1}$ & 4 & $x_{4}$ & $y_{4}$ \\
$\ldots$ & $\ldots$ & $\ldots$ & $\ldots$ \\
$O_{2}$ & 5 & $x_{5}$ & $y_{5}$ \\
$O_{2}$ & 6 & $x_{6}$ & $y_{6}$ \\
$O_{2}$ & 7 & $x_{7}$ & $y_{7}$ \\
$\ldots$ & $\ldots$ & $\ldots$ & $\ldots$ \\
$O_{3}$ & 4 & $x_{5}$ & $y_{5}$ \\
$O_{3}$ & 5 & $x_{8}$ & $y_{8}$ \\
$O_{3}$ & 6 & $x_{9}$ & $y_{9}$ \\
$\ldots$ & $\ldots$ & $\ldots$ & $\ldots$ \\
\hline
\end{tabular}

\begin{tabular}{|c|c|c|c|}
\hline$O_{i d}$ & $g_{i d}$ & $t_{s}$ & $t_{f}$ \\
\hline$O_{1}$ & $H_{1}$ & 1 & 10 \\
$O_{1}$ & $C$ & 20 & 30 \\
$O_{1}$ & $P$ & 50 & 70 \\
$O_{1}$ & $H_{1}$ & 100 & 140 \\
$O_{2}$ & $H_{2}$ & 5 & 20 \\
$O_{2}$ & $C$ & 25 & 40 \\
$O_{2}$ & $P$ & 50 & 80 \\
$O_{2}$ & $H_{2}$ & 120 & 140 \\
$O_{3}$ & $H_{2}$ & 4 & 10 \\
$O_{3}$ & $P$ & 15 & 40 \\
$O_{3}$ & $H_{2}$ & 60 & 140 \\
\hline
\end{tabular}

Figure 1: Introductory example (left), raw trajectories (center), and PoIsbased trajectory (right).

interesting patterns in the trajectory data, or be interested in queries like "Give the percentage of trajectories visiting two restaurants in the same day."

A typical way of performing trajectory analysis is to store trajectory data in relational databases for example, in repositories called trajectory data warehouses Vaisman and Zimányi2013, Leonardi et al.2014, over which online analytical processing (OLAP) is performed. OLAP refers to a collection of operations for exploiting multidimensional databases (MD). In a MD database, data are perceived as data cubes, such that the axes in these cubes are called dimensions, and cells in the cubes contain one or more measures that quantify facts. Dimensions are further organised in aggregation hierarchies, thus measures can be aggregated along them. Queries in OLAP consist in sequences of operations that manipulate the data cube. The most usual of these operations allow aggregating and disaggregating measure values in the cube cells along the dimensions (Roll-Up and Drill-Down operations, respectively); selecting a portion of the cube (Dice); or projecting the data cube over some of its dimensions (Slice). A problem with this approach, particularly with the huge volumes of data available nowadays, is the "impedance mismatch" between the way in which data are modelled and stored. Given that trajectories can be typically considered as graphs, storing trajectory data as relations may seem unnatural, since current database technology provides solutions that allow storing graphs in native form, as explained next. Thus, this paper discusses how this OLAP-style semantic trajectory analysis can be performed over graph databases.

Property graphs [Robinson et al.2013] (graphs whose nodes and edges 
are annotated with properties), are typically used to model networks (e.g., social networks, sensor networks) to perform data analysis. The property graph data model is an abstraction that can be used to represent trajectories, either in their raw or semantic forms. In this model, for example, the point coordinates or the PoIs can be represented as nodes, and there is an edge from one point (or PoI) to the consecutive one in the sequence of points (or PoIs) in the trajectory. In addition, spatio-temporal coordinates can be also included as properties, as well as other characteristics of the visited places. Also, hierarchical contextual data can be defined, which would allow representing the trajectory graph at different granularities, leading to the notion of trajectory aggregation. With these machinery, a data scientist may also perform OLAP-like analysis over trajectory graphs. Several different graph data models for this (called Graph OLAP in the remainder) can be found in the literature, and are discussed in Section 2, Modelling trajectories using graphs allows storing them in native form (i.e., as graphs) using graph databases Angles2012, Angles2018, rather than in relational databases, thus preventing the "impedance mismatch" problem mentioned above. In particular, in this paper, the graph database software Neo4 $]^{1}$ is used. Besides its popularity, the Neo4j community has developed several libraries of functions; one of these is a spatial library ${ }^{2}$, which allows defining different spatial layers, that can enhance the analysis possibilities.

Surprisingly, a careful analysis of the literature reveals that there is almost no work on the subject, in light of which this paper aims at answering the following questions: can graph databases be successfully used to model, store, and query semantic trajectory data? If so, which are the kinds of queries that could benefit the most from this approach? In particular, the paper focuses on analytical queries, that typically require aggregating the trajectory graph up to different granularity levels. In addition, the paper aims at showing that using graph databases has several advantages over the typical solution of storing semantic trajectory data in relational databases, based on the following assumptions and facts. First, as already mentioned, graphs are a natural way to represent trajectories. Second, storing trajectories in a graph database allows having a powerful machinery of algorithms to exploit these data, at no extra cost. Ad-hoc alternatives require using different tools for different problems (e.g., querying, finding patterns). Third, graph databases like Neo4j include a high-level graph query language (in the case of Neo4j, Cypher), together with a large collection of functions included in plugins that are easily added to the database, while the relational solution requires the use of different languages for the different tasks. Fi-

1 http://www.neo4j.com

2 https://neo4j-contrib.github.io/spatial/ 
nally, expressing queries using graph query languages is far more intuitive for a non-expert user, than doing this through, for example, complex SQL queries.

A real-world running example is used for this study, based on the "Foursquare New York" dataset 3 This dataset includes about ten months of check-in data in New York City, collected from the Foursquare social network. In addition, a Time dimension hierarchy, and a Stop dimension hierarchy are defined as contextual information. To make the analysis more interesting, the dataset is enriched with geographic New York City data 4 It is worth noting that Foursquare is a Location-Based Social Network (LBSN), therefore check-ins in this dataset do not conform typical GPS-based trajectories. However, taking into account the goals of this research, these sequences of check-ins can be considered as semantic trajectories where stops have no duration, and there is no move between these stops (or, equivalently, it can be assumed that a "move" occurs between two zero-duration stops).

In summary, to address the research questions above, the following tasks are performed:

1. A property graph data model for representing semantic trajectories. This model is based on the family of Graph OLAP modelling techniques to favour aggregation of trajectory data represented as graphs.

2. The model is applied to the "Foursquare New York" dataset, enriched with dimensional contextual information and spatial data, and implemented over a Neo4j database. In addition, the trajectory database is implemented on a PostgresSQL relational database.

3. A collection of twelve analytical queries, classified into five classes, is defined. These queries are written in the Cypher query language over the Neo4j implementation and in SQL over the relational implementation.

4. The queries are run and the results discussed.

As it will be explained, the study shows that most of the hypotheses are verified. Queries in the graph-based trajectory model are not only more natural to express than in the relational alternative, but also show a better performance (ranging from 1.2 to 7 times faster) than the latter in three of the five classes of queries studied, particularly when sequential patterns are looked for (e.g., in queries like "Find trajectories that go directly from a home to a station and then to an airport"), and when aggregation is involved

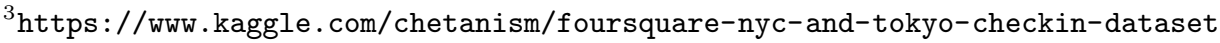

${ }^{4}$ Maps where downloaded from http://www.mapcruzin.com
} 
(for example "Compute the total length of each trajectory, as the sum of the distance between each pair of consecutive stops").

The remainder of this paper is organised as follows: in Section 2 related work is discussed. Section 3 very briefly presents some basic notions on trajectories and OLAP on graphs. Section 4 introduces the data model, while in Section 5 a case study is discussed. Section 6 concludes the paper, addressing future work and open problems.

\section{Related Work}

The field of moving object databases (MODs) has been extensively studied by the GIS community. The interested reader is referred to Güting and Schneider Güting and Schneider2005 for a survey of this large area. Several techniques for the semantic annotation of trajectory data have been proposed and studied. Mouza and Rigaux du Mouza and Rigaux2005 propose a model in which raw trajectory data are transformed in a sequence of moves (zones represented by labels or IDs). They also define a regular expression-based query language that allows querying for mobility patterns. Giannotti et al. Giannotti et al.2007 introduce Temporally Annotated Sequences (TAS) as a basis for trajectory pattern mining. A trajectory pattern is defined as a collection of trajectories that visit the same places in sequence with similar time gaps between each of these places. The concept of a Region of Interest (RoI) dynamically computed from the trajectories is defined. With a similar idea, Spaccapietra et al. Spaccapietra et al.2008 define "stops and moves" to semantically enrich trajectories. Alvares et al. Alvares et al.2007] study trajectory analysis based on the concepts of stops and moves. The concept of stop here differs from the notion of RoI: the former is application-dependant, defined in advance and really relevant to a trajectory, while the latter is detected dynamically. Finally, Gómez and Vaisman Gómez and Vaisman2013] presented RE-Spam, a language for discovering sequential patterns in semantic trajectories, based on reg-

ular expressions. The work by Parent et al. Parent et al.2013. provides a comprehensive description of the notions of trajectory and semantic trajectory.

Regarding graph databases, two database models are used in practice:

(a) Models based on RDF $5^{5}$ oriented to the Semantic Web.

(b) Models based on property graphs.

\footnotetext{
5 https://www.w3.org/RDF/
} 
Models of type (a) represent data as sets of triples where each triple consists of three elements that are referred to as the subject, the predicate, and the object of the triple. These triples allow describing arbitrary objects in terms of their attributes and their relationships to other objects. Informally, a collection of RDF triples is an RDF graph. In models of type (b) Angles et al.2017, nodes and edges are labelled with a sequence of attribute-value pairs. It is an extension of classical graph database models, frequently used for implementations in practical applications. The main reason for storing attributes in nodes and edges is speeding up the retrieval of the data directly related to a certain node. For an extensive and comprehensive bibliography on graph database models, the interested reader is referred to Angles and Gutierrez2008, Angles2018. Although the models in (a) have a general scope, the structure of RDF makes them not as efficient as the other models, which are aimed at reaching a local scope. An important feature of RDF-base graph models, however, is that they follow a standard, which is not yet the case for the other graph databases, therefore they are typically used for metadata representation. Therefore, many works have proposed RDF to annotate trajectories with semantic information [Fileto et al.2015, da Silva et al.2015, Ruback et al.2016]. Hartig Hartig2014 proposes a formal way of reconciling both models formally, through a collection of well-defined transformations between property graphs and RDF graphs. He shows that property graphs could, in the end, be queried using SPARQL $\sqrt[6]{6}$ the standard query language for the Semantic Web. The model used in the next sections to represent and query trajectory data is based on the concept of property graphs.

Several data models to perform OLAP Kimball1996] on graphs have been proposed. GraphOLAP [Chen et al.2009], conceptually, is a framework for OLAP on a set of homogeneous graphs, based on splitting the graph into a collection of snapshots that are aggregated in two ways, called Informational and Topological OLAP aggregations. GraphCube [Zhao et al.2011] provides a framework for computation and analysis on OLAP cubes using the different levels of aggregation of a graph. This framework introduced the notion of cuboids. A recent proposal, denoted Graph OLAP Gómez et al.2017] models the problem as basic graph data (at the finest granularity defined for the application), background information in the form of dimension hierarchies, and a collection of so-called graphoids (the basic graph aggregated at different granularity levels defined by the background dimensions. Analogously to the models commented above, the classic OLAP operations (Rollup, Slice, Dice, Drill-down) are also defined in terms of the components of the model.

\footnotetext{
6 https://www .w3.org/TR/rdf-sparql-query/
} 
Regarding the use of graph databases for analyzing trajectory data, Gryllakis et al. Gryllakis et al.2018 implemented over Neo4j, the Hermes MOD originally developed over Oracle Pelekis et al.2015]. This is basically a datatype system for representing semantic trajectories. The authors also extended the Neo4j spatial plugin to facilitate operations on semantic trajectories. In particular, the authors address the problem of answering what they call spatio-temporal-keyword pattern (STKP) queries Gryllakis et al.2017. Queries of this kind ask for episodes satisfying a pattern which may include keywords, spatial and temporal conditions. For example, a STKP query may ask for trajectories starting with an episode (basically a stop) whose geometry is contained in a certain bounding box, followed by an indefinite sequence of episodes.

As far as the authors of this work are aware of, the only work discussed above that somehow compares with the one presented here, is the work by Gryllakis et al. Gryllakis et al.2018. However, such work is aimed at extending Hermes with datatypes, and the Neo4j spatial plugin, to answer an specific kind of queries. The work in the present paper focuses in modelling and storing semantic trajectories as graphs, in order to support analytical (OLAP) queries of different kinds, topics not addressed elsewhere. This approach can therefore be applied to any graph database, although in this paper Neo4j is used, with no addition whatsoever.

\section{Background}

A short background on the topics addressed in this paper is given next, to make the paper self-contained. Basic notions on trajectories are introduced first. The second part of this section briefly presents the Graph OLAP data model that will be used in the remainder.

\subsection{Trajectories and Semantic Trajectories}

The definitions next formalise the intuitive notions given in the example of Section 1. The notion of "trajectory" is defined first.

Definition $1 A$ trajectory is a sequence $\left\langle\left(t_{0}, x_{0}, y_{0}\right),\left(t_{1}, x_{1}, y_{1}\right), \ldots,\left(t_{N}, x_{N}\right.\right.$, $\left.\left.y_{N}\right)\right\rangle$ of spatio-temporal points, where, for $i=0, \ldots, N, t_{i}, x_{i}, y_{i} \in \mathbf{R}$. There order $t_{0}<t_{1}<\cdots<t_{N}$ induces a natural order on the time-space points in the trajectory. The time domain of the trajectory is the interval $\left[t_{0}, t_{N}\right]$.

A table like the one in Figure 1 (center) is called a Moving Object Table (MOT). In practice, MOTs can contain huge amounts of data. Thus, 
querying raw trajectory data may be extremely time consuming. Furthermore, data scientists are often not concerned with such level of geometric detail, and they rather look for more aggregated information. Also, answering queries may require semantic information that is not present in the MOT. As a solution, the literature in the field proposes using the notion of stops and moves to reduce the size of the MOT. Thus, a trajectory can be represented in terms of so-called places of interest for a particular application, characterized as what are denoted stops. This concise table (see Figure 1 (right)) cannot encode the complete information contained in the MOT. However, it allows to quickly access information of interest without having to consult the complete data set. For this, the notion of "Place of Interest of an Application" must be defined first [Alvares et al.2007.

Definition $2 A$ place of interest (PoI) $C$ is represented by a tuple $\left(R_{C}, \Delta_{C}\right)$. The geometrical figure $R_{C}$ is a (topologically closed) polygon, polyline or point in the plane $\mathbf{R}^{2}$, called the geometry of $C$, and $\Delta_{C}$ is called the minimum duration of $C$.

Given an application $\mathcal{A}$, the places of interest of $\mathcal{A}$, denoted $\mathcal{P}_{\mathcal{A}}$, is a finite collection of PoIs (relevant to this application) whose geometries mutually do not intersect.

Definition 3 Let $T=\left\langle\left(t_{0}, x_{0}, y_{0}\right),\left(t_{1}, x_{1}, y_{1}\right), \ldots,\left(t_{n}, x_{n}, y_{n}\right)\right\rangle$ be a trajectory and let $\mathcal{P}_{\mathcal{A}}=\left\{C_{1}=\left(R_{C_{1}}, \Delta_{C_{1}}\right), \ldots, C_{N}=\left(R_{C_{N}}, \Delta_{C_{N}}\right)\right\}$ be the places of interest of an application $\mathcal{A}$. A stop of $T$ with respect to $\mathcal{P}_{\mathcal{A}}$ is a contiguous sub-trajectory $\left\langle\left(t_{i}, x_{i}, y_{i}\right),\left(t_{i+1}, x_{i+1}, y_{i+1}\right), \ldots,\left(t_{i+\ell}, x_{i+\ell}, y_{i+\ell}\right)\right\rangle$ of $T$ of maximal size such that for some $k \in\{1, \ldots, N\}$ the following holds: (a) $\left(x_{i+j}, y_{i+j}\right) \in R_{C_{k}}$, for $j=0,1, \ldots, \ell$; (b) $t_{i+\ell}-t_{i}>\Delta_{C_{k}}$. That means, if the user stays more than the $\Delta$ threshold, the place is considered a stop. A move of $T$ with respect to $\mathcal{P}_{\mathcal{A}}$ is a maximal contiguous subtrajectory of $T$ :

(a) in between two temporally consecutive stops of $T$;

(b) between the starting point and the first stop of $T$;

(c) between the last stop of $T$ and ending point of $T$;

or the trajectory $T$ itself, if $T$ has no stops.

There are many possible variations of the definition of stops and moves of a trajectory, depending for instance, on the interpolation technique used on the trajectory samples, or in the tolerance used to consider whether an object is inside or outside a place of interest [Parent et al.2013, Spaccapietra et al.2013. Discussing these alternatives is outside the scope of this paper. Intuitively, semantic trajectories are produced replacing a sequence of $\langle t, x, y\rangle$-tuples by a sequence of stops, taken from the collection of PoIs. More formally: 
Definition 4 A semantic trajectory is a trajectory (see Definition 1) with added semantic annotations. Formally, a semantic trajectory is a structure of the form $\mathcal{T}_{s}\left(O_{i d}, \mathcal{S}\right)$, where $O_{i d}$ is a moving object identifier, and $\mathcal{S}$ is a sequence of pairs of the form $\left(s_{i}, L_{i}\right)$, where $s_{i}$ and $L_{i}$ are defined as follows: $s_{i}$ is a stop (Definition (3) traversed by $O_{i d} ; L_{i}$ is a list of pairs (metadata, value), where metadata is an attribute representing a characteristic of $s_{i}$ and value is the value of such attribute for $s_{i}$. Moreover, $\mathcal{S}$ is time-ordered, that means, the $s_{i}$ 's are listed in the order they were traversed by the object with $O_{i d}$ in $\mathcal{T}_{s}$.

\subsection{Graph OLAP}

The model adopted in this paper for representing trajectories as graphs, is composed of three main types of elements. The first type is a collection of OLAP dimension hierarchies that represent contextual (or background) information for the graph (trajectory) data. Dimensions have schema and instances, as usual in databases. A dimension schema is a lattice with a unique top and a unique bottom. Each node in a dimension schema is called a dimension level, which, in turn, is associated with level instances, containing elements of a certain domain. Dimension instances are collections of hierarchies. The second element in the model is the base graphoid. Assuming a collection of dimensions $D_{1}, \ldots, D_{d}$ in a certain application domain, the nodes and edges of the base graphoid are defined at the bottom levels of the background dimensions (i.e., $D_{1}, \ldots, D_{d}$ ). The nodes and edges in a graphoid have a type, associated with the corresponding background dimensions. The third type of element in the model are the graphoids defined at different levels of granularity (these levels are defined in the background dimensions).

Figure 2 illustrates the above for the running example (the complete example is detailed in Section 4 and depicted in Figure 4). The base graphoid, representing the trajectory data, is shown in the upper part of the figure. Here, the Stop nodes are linked by edges labelled \#trajstep. Background dimension hierarchies for the Stop and Time dimensions are defined as Stop $\rightarrow$ Venue $\rightarrow$ Categories $\rightarrow$ Category, and Minute $\rightarrow$ Hour $\rightarrow$ Day $\rightarrow$ Month $\rightarrow$ Year hierarchies, respectively. The base graphoid is defined at the Stop and Instant granularity levels of these dimensions. Other trajectory graphoids can be defined at different dimension levels, climbing along the Stop and Time hierarchies. For example, a trajectory graphoid denoted a (Stops.Category, Time.Datehour)-graphoid is the base graphoid aggregated at the [\#Datehour] and [\#Category] levels, and it is depicted in the bottom part of Figure 2.

Over this model, a collection of operations are defined, analogously to 


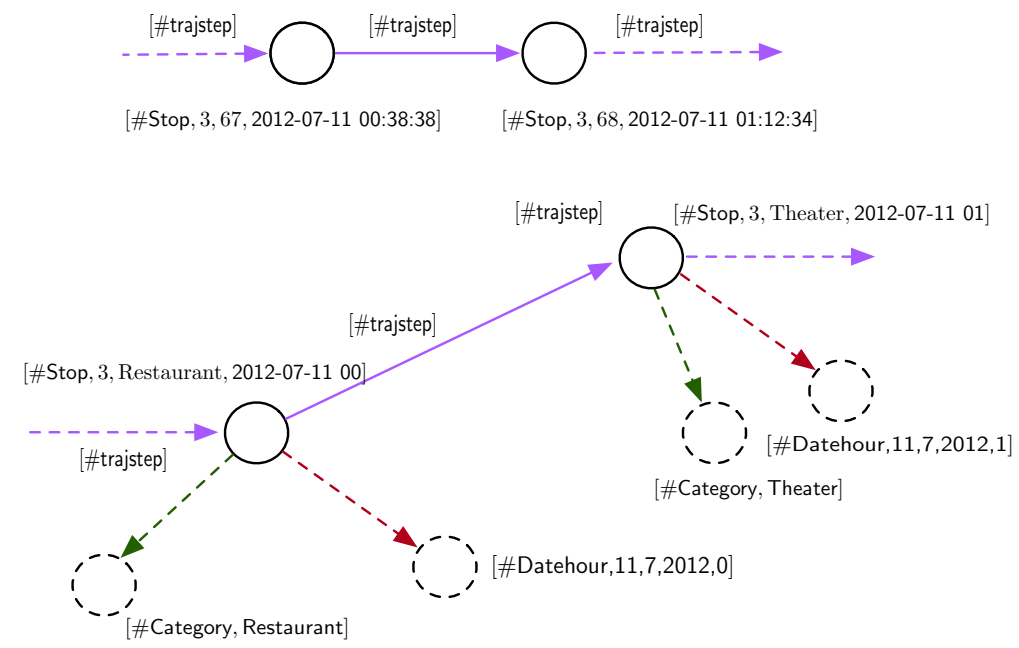

Figure 2: Base graphoid and (Stops.Category, Time.Datehour)-graphoid for the trajectory graph in the running example.

the classic OLAP operations that are performed over multidimensional data cubes. This way, the Climb operation replaces each node in a graphoid with the corresponding node according to the associated dimension level. For example, if an attribute of a node representing trajectory stops (as in Figure 2 is instant, a climbing operation to the Hour level along dimension Time, will produce the (Time.Datehour)-graphoid. Other operations are defined over the graph model resembling the corresponding operations on cubes. Operation Roll-Up takes a graphoid to a coarser granularity level along a dimension $D_{d}$ up to a level $\ell_{u p}$, and performs an aggregation over a collection of measures. The Drill-Down operation does the opposite of Roll-Up, taking a graphoid to a finer granularity level, along a dimension $D_{d}$, down to a level $\ell_{\text {down }}$. The Dice operation produces a subgraphoid of another one, whose nodes satisfy a Boolean condition $\varphi$ defined over a elements in the graphoid model. All these operations will be further explained over the running example in Section 5 .

\section{Representing and Storing a Trajectory as a Graph}

This section shows, through an example, how a trajectory can be represented using the property graph data model, and loaded into a graph database. First, the running example to be used in the remainder of the paper is presented. Then, this example is modelled as a trajectory graph. 


\subsection{Running Example}

As mentioned in Section 1, the running example considers data from the "Foursquare New York" dataset. This dataset includes about ten months of check-in data in New York City, with a total of 227,428 check-ins collected from the Foursquare social network. When someone checks-in into some place (an airport, a restaurant, her private home, for instance), a point is recorded. Data from more than a thousand users are stored in this dataset. The dataset is used to analyse how people move in the city. Further, the dataset is enriched by adding spatial and not-spatial contextual information to the PoIs. Such information is organised as aggregation hierarchies, so semantic trajectories can be represented at different granularity levels. For example, in the dataset, an $(x, y)$ point is represented as a (zero duration-) stop by means of a (latitude, longitude) pair. This stop can be a train station, which in turn is categorised as "Station". The next stop may be a Thai restaurant, which, at a coarser level, is categorised as "Restaurant". Thus, this sub-trajectory becomes a sequence of the form 〈...Station, Restaurant, ... >. A temporal hierarchy is also used for analysis. For example, a check-in into a PoI at 3pm can be aggregated as an "afternoon" stop, if the analyst is not interested in a finer level of detail. Note that the original dataset contains the check-in data, and the data enrichment has been performed to enhance this use case, as it is explained below.

Many interesting queries of different types can be posed over the scenario described above. For example, since check-ins in this dataset also include public transport, queries like the following can be expressed.

- "List the users moving between places of interest using a taxi."

- "Which users travel to an airport by taxi during the night."

The second query implies an aggregation along the temporal dimension. As another type of problem, interesting patterns may be investigated, like, for instance:

- "Return the trajectories in which users go from their homes to an airport after 5pm."

- "Give the percentage of trajectories in which users go from a restaurant to a sport event and end at a coffee shop."

The addition of spatial data layers allows queries like the following ones.

- "Compute the number of users moving between two or more boroughs in the same day" 
- "Compute the average distance travelled per user and per day"

Accounting for all of the above, the graph trajectory model is presented next.

\subsection{Modelling the Trajectory Graph}

The "Foursquare New York" dataset contains check-in data of 1083 users into different places, or "venues". This comprises 1083 trajectories of different lengths. For this study, long trajectories are not split into smaller ones in a "preparatory phase". In any case, this could be done through queries, asking for example, for places visited by a user on a certain day. Each row in the dataset contains the following information: the user identifier, denoted userld in the table below; the identifier of the place where the user checkedin, called venueld; the categorization of the venue, with the identifier and the category name, called, respectively, venueCatld and venueCat (these describe the kind of venue, e.g., private home, Thai restaurant, etc.); the geographic coordinates of the stops, denoted latitude and longitude; the timezone offset, called t-zone in the table; the timestamp for the stop, called timestamp (representing the check-in time for the user at the stop). These data where loaded into a Postgres relational database and an additional field was added, indicating the relative position of the stop in the trajectory, called pos. The resulting relational schema and an example tuple are depicted next.

The Trajectories table has the form:

\begin{tabular}{|c|c|c|c|c|c|c|c|c|}
\hline userId & venueId & venueCatId & venueCat & lat. & long. & t-zone & timestamp & pos \\
\hline 1 & $4 a b c 1 \ldots$ & 4 bf5 ... & Seafood Rest. & $40.78 .$. & $-73.97 .$. & -240 & $2012-04-0423: 31: 31$ & 1 \\
\hline$\ldots$ & $\ldots$ & $\ldots$ & $\ldots$ & $\ldots$ & $\ldots$ & $\ldots$ & $\ldots$ & $\ldots$ \\
\hline
\end{tabular}

There is also a table Categories, not in the dataset. This table has been included in order to make the case study more interesting, and to add semantics to the trajectory data. The idea is to further categorize venues. For example, in the table Trajectories, attribute venueCat, in the first row details the kind of venue. Thus, a category type 'Restaurant' can be defined, to aggregate data at a coarser level of detail. Actually, this represents a level in an aggregation hierarchy. This new aggregation level is materialized by the attribute catType in the table Categories below. This categorization been produced manually, analyzing all kinds of venues one by one, and assigning to them a category type. All in all, thirty-three category types have been defined. The dataset defines 251 venue categories, and each one of them has been assigned to one of category type. In summary, 38,333 venues are classified into 251 categories, which in turn are classified into 33 category types. 


\begin{tabular}{|c|c|c|c|}
\hline venueId & venueCat & venueCatId & catType \\
\hline 4aa06479f964a5.. & Pizza Place & 4bf58dd ... & Restaurant \\
\hline$\ldots$ & $\ldots$ & $\ldots$ & $\ldots$ \\
\hline
\end{tabular}

To model semantic trajectory data as a graph, with graph aggregation in mind, the paper adopts the Graph OLAP model described in Section 3.2. The base graphoid is composed of the trajectories themselves. Each node in the base graphoid represents a stop in the trajectory, and has properties (attributes) userid (i.e., the trajectory id), instant (the time instant when reaching the stop), and position (the relative position of the stop in the trajectory). Note that, like in any conceptual design, many modelling options can be considered (for example, regarding the representation of an object as a node or as an attribute, or placing an attribute in a node or in a relationship). The one chosen for this use case is only one of them.

In order to analyse trajectories along contextual dimensions (called background dimensions in Graph OLAP), and at different levels of granularity, these dimensions must be built and associated with the base graphoid (in what follows, the "trajectory graph"). In this case, hierarchies for Stop and Time dimensions are built. For the former, fields in the original dataset are used, together with the categories defined for each kind of venue. The latter is built using software libraries (as explained below).

The Time hierarchy aggregates data from the instant represented in the stops, up to the year level as follows (this is a conceptual representation, the actual one is described below):

$$
\text { Minute } \rightarrow \text { Hour } \rightarrow \text { Day } \rightarrow \text { Month } \rightarrow \text { Mear }
$$

The background dimension for stops is defined as follows. The bottom level of the hierarchy, that is, Stops (represented in the trajectory graph), is associated with the level Venue, which in turn is associated with level Categories through a relationship denoted hasCategory (the category of the venue is included in the dataset, and denoted venueCat in the table above). The hierarchy is completed with the level Category such that there is an m:1 relationship, denoted isSubcategoryOf, from Categories to Category. The instance of the level Category corresponds to the attribute catType in the table Categories. For example, the element Afghan Restaurant at the Categories level, is associated with the element Restaurant at the level Category. Thus, the Stop dimension hierarchy is (conceptually) of the form

$$
\text { Stop } \rightarrow \text { Venue } \rightarrow \text { Categories } \rightarrow \text { Category. }
$$

As a remark, note that although the name Categories for the level may seem confusing, the decision of keeping it (instead of, for example, subCategories), is led by the intention to remain faithful to the original data. 


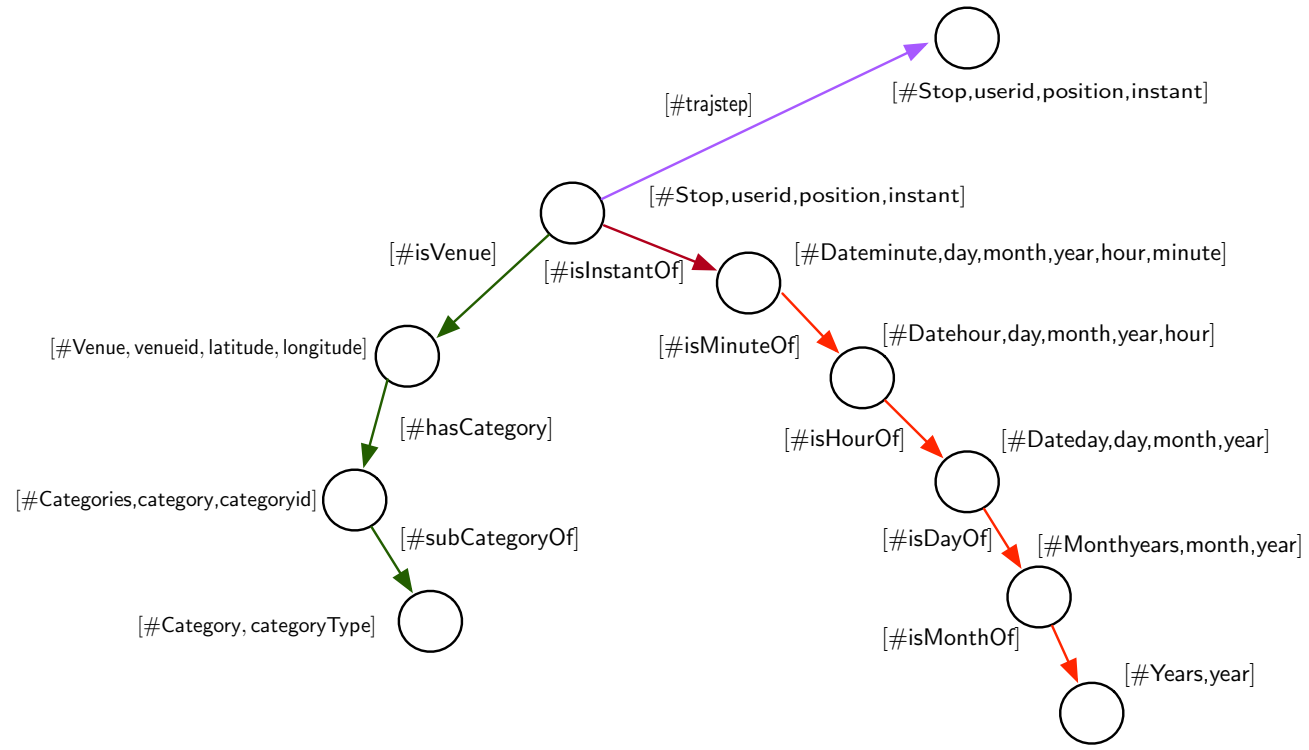

Figure 3: Schema of the trajectory database.

Figure 3 depicts the schema of the trajectory database expanded with the background dimensions, using the Graph OLAP notation. Stops in each trajectory are represented by a node of type \#Stops, with its corresponding attributes. Between each pair of stops there is an edge labelled trajstep. The figure also shows the schema of the background dimensions. Each aggregation level in the dimension hierarchies is shown as a tuple containing the node type and its corresponding level attributes. For example, in the Stop dimension, the level Venues is represented as the tuple [\#Venue, venueid, latitude, longitude]. The relationships representing the hierarchy for the venues are indicated by the edges labelled [\#isVenue], [\#hasCategory], and [\#subcategoryOf]. The Time dimension is represented analogously. Note that, although is this case the relationships do not contain attributes, in general this will not be the case.

Figure 5 shows a portion of an instance of the trajectory graph, represented as a property graph using the Graph OLAP notation. Two trajectories are shown, namely for user ids 2 and 3 . At the instance level, there is an edge between two stops $s_{1}$ and $s_{2}$ if $s_{2}$ is the stop occurring immediately after $s_{1}$. For the latter trajectory, the complete instance of the hierarchy for the stop in position 67 is shown. It can be seen that, for each node attribute in the schema, there is a value in the instance. Also, note that although is this case data are quite structured, one feature of a graph data model is that 


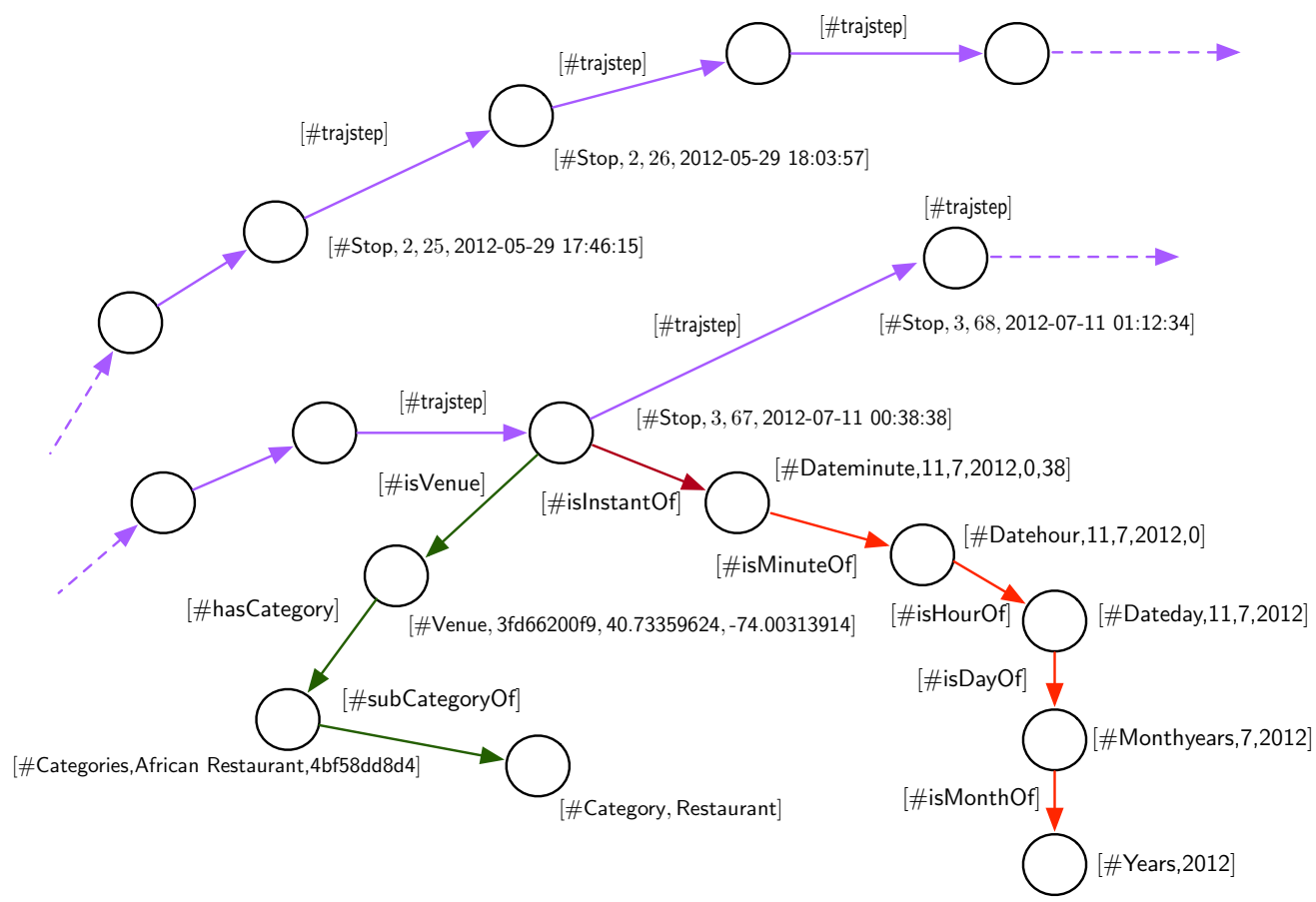

Figure 4: Portion of the trajectory graph instance.

the schema is totally flexible and unstructured, meaning that, for example, it is not a requirement that all nodes contain the same attributes.

\subsection{Storing the Trajectory Graph}

The last step of this process consists in loading the trajectory graph into the Neo4j graph, which is quite straightforward, and therefore details are omitted here for the sake of brevity. To build the Time dimension, functions in the APOC library for Neo4j $]^{7}$ are used. A portion of the resulting graph is shown in Figure 4 (the attributes are not shown, because of the graph interface). The edges between stops are highlighted in bold font. The Time hierarchy is not shown for the sake of clarity. It can be seen, for example, that node 86 corresponds to the venue with id 11943, which in turn is a vegetarian restaurant, further classified as a restaurant. The path between nodes 84 through 87 is also shown, with edges labelled trajstep.

In addition, three spatial layers are defined (not shown in the figure), namely nycdistricts, new_york_highway, and nyorkpois, representing, respec-

\footnotetext{
${ }^{7}$ https://neo4j.com/developer/apoc/
} 
tively, the districts, highways and places of interest in New York City. These layers were imported from public data, and loaded into the Neo4j database.

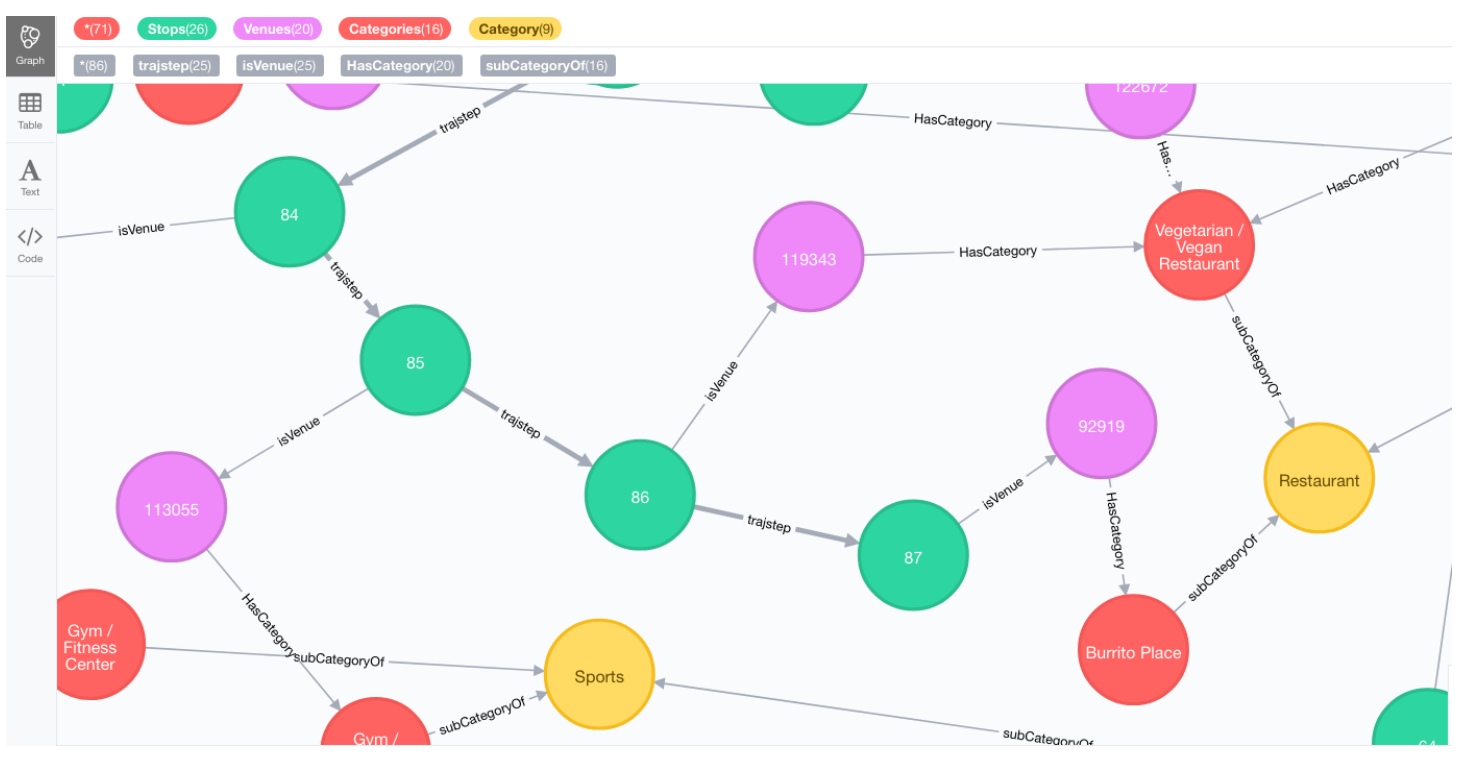

Figure 5: The trajectory graph in a Neo4j database

The trajectory graph is now ready to be exploited with the Neo4j tools, and queried using the high-level language Cypher. This is addressed in the next section.

\section{A Case Study}

The trajectory graph described in Section 4 can be analysed in many ways. The first part of this section presents a collection of queries classified into five query types. Note that the model is aimed at addressing analytical queries, which take advantage of the contextual dimensions of the Graph OLAP data model used here, in order to study semantic trajectories at different granularity levels. Thus, most queries include climbing and aggregating data along the dimension hierarchies, and the operations á la OLAP involved are explained for each query. Queries are expressed in the Cypher query language, the high-level language that comes with Neo4j. Only the Cypher expressions which may be intuitively understood even by non-expert readers, have been included. The remaining queries are detailed in the appendix. The second part of the section discusses the performance of the queries, and compares it (when possible) against the analogous relational ones. 


\subsection{Analytical Semantic Trajectory Queries}

This section addresses queries aimed at helping in the decision-making process, for example, for transport policy makers, companies considering starting a new business, etc. Queries are organized into classes that account for their main characteristics.

Queries computing non-recursive patterns These are typical queries in trajectory analysis. Below, an example of a query asking for a simple pattern is presented. Complex patterns are commented later on, when studying transitive closure queries. The Graph OLAP model allows finding patterns at different granularity levels. In this case, note that the semantic trajectory graph is defined at the granularity of the stops, and the query below works at the Category level.

Query 1 Find the trajectories that go from a private home to a station and then to an airport, without intermediate stops.

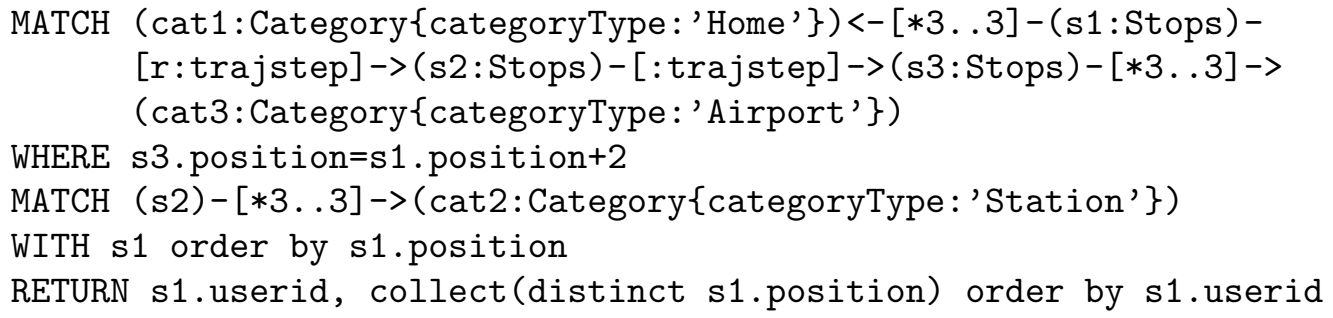

The first MATCH clause describes a pattern matching a sequence of three consecutive stops; the first and last ones include OLAP Climb operations along the Stops dimension up to the Category dimension level (the coarser level in the hierarchy). The second MATCH describes the same climbing pattern for the intermediate stop. At the Category level, a Dice operation is performed, keeping the desired category, such that only the required 3-stop patterns are kept. The WITH clause before the last line acts like a 'pipe', which passes variables from one portion of the code to the next one (in this case, it passes s1). The result is given as pairs of the form (userld, $\langle\mathrm{LOP}\rangle$ ), where userld identifies the trajectory, and LOP is a list of the initial position of each pattern, within the trajectory. Note that in the climbings a shorthand is used, since there is only one possible path up to the Category level, and no variables are needed over any intermediate level. If the climbing path were needed, the second MATCH clause would read:

(s2)-->(:Venues)-[:HasCategory]->(:Categories)-[:subCategory0f]-> (cat2: Category\{categoryType: 'Station'\}) 
The next query aims at comparing performance for a longer pattern.

Query 2 Find the trajectories that go from a bar (or similar) to a restaurant, again to a bar (or similar) and end at a restaurant, without intermediate stops.

The Cypher expression for this query is given in the appendix (Query 2), and it is further discussed in Section 5.3 .

Queries computing travelled distance Queries in this class compute the distance between points, but they do not use spatial layers or special libraries. The functions are part of the Cypher language.

Query 3 For each trajectory, compute the distance travelled between each pair of consecutive stops.

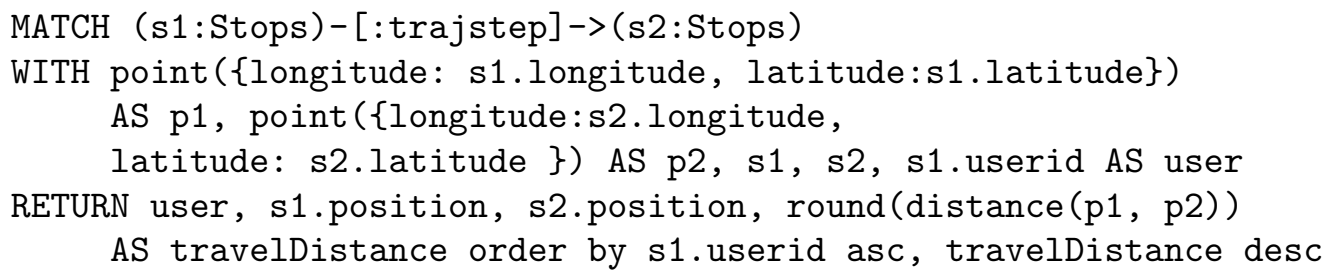

Here, all consecutive pairs of stops are computed first (by pattern matching, rather than joins, which would be the case in the relational model). Then, the (latitude, longitude) pairs of each stop are obtained. Finally, for each trajectory, the distance between two consecutive stops is computed.

The next query shows how to easily analyse distances travelled between different kinds of places, combining characteristics of Queries 1 and 3 and including aggregation along background dimensions.

Query 4 For all trajectories that go directly from a private home to an airport, list the user identifier, together with the distance travelled between these two places each time that this pattern occurs.

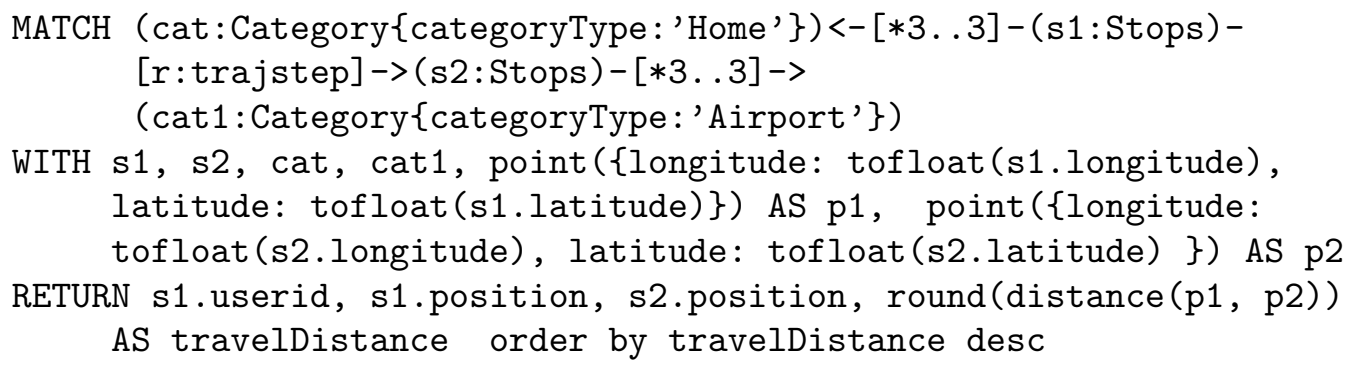


It can be seen that this query first performs two climbings up to the Category level to select (Dice) only stops corresponding to homes and airports. Then, the aggregation is computed at the bottom level of the dimension (that is, it first climbs, and then computes the distance at the Stop level).

Spatial trajectory queries These queries make use of the spatial information contained in layers other than the one where the trajectories are represented. The query below uses the layers containing the places of interest ("nyorkpois"), and calls the function withinDistance contained in the Neo4j spatial library.

Query 5 Find the trajectories passing at less than $100 \mathrm{~m}$ of a public school.

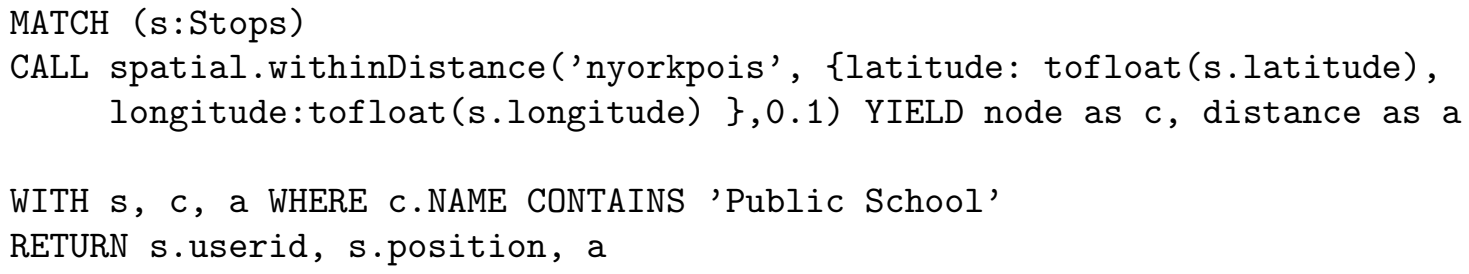

This query uses the NAME feature included in the spatial layer. The spatial.withinDistance function computes the distance between stops in the trajectories and the PoIs in the spatial layer. The query returns for each user (trajectory), the user identifier, the position of the stop in the trajectory, and the distance between the stop and the school.

The following query is more general, since it does not ask for a particular kind of PoI.

Query 6 List the trajectories starting at less than 300m from a Place of Interest of the city, returning the trajectory id (i.e., userld), and the actual distance, for all the PoIs in the answer.

The Cypher expression is given in Query 6 of the appendix.

Aggregation queries Queries in this class include different forms of aggregations, typical in data analytics.

Query 7 Compute the number of different categories of venues visited by month.

MATCH $p=($ s1:Stops $)-[* 3 . .3]->$ (scat: Category)

WITH $p, s 1$, scat 


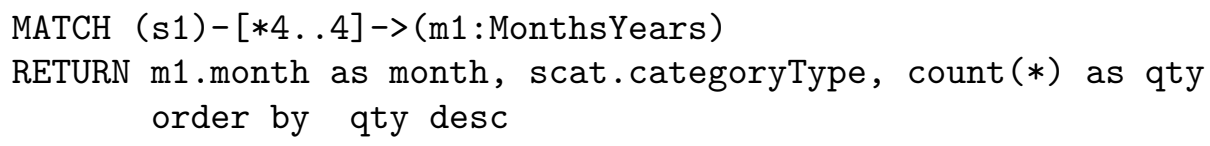

Here, two climbings are required: one along the Stop dimension hierarchy up to the Category level, and another one along the Time dimension up to the MonthsYears level. Finally, the aggregation is performed. The climbing and the aggregation conform a Roll-up operation.

Two more examples of aggregate queries are given next.

Query 8 Compute the number of stops per day per user, along with the starting position of each sub-trajectory for each day.

Query 9 For each trajectory, compute its total length, as the sum of the distances between each pair of stops.

The Cypher expressions for these queries are given in Queries 8 and 9 of the appendix. The queries are also discussed in Section 5.3 .

Transitive closure queries This type of queries are quite more complex than the previous ones, since they do not only include Graph OLAP operations or pattern to match, but also require computing the transitive closure of the trajectory graph. In the sequel, the main idea is explained. However, given that the understanding the details may be hard for readers not familiar with Cypher, most technical details are given in the appendix.

Query 10 For each trajectory, find the paths that go from a private home to an airport in the same day.

This query requires some explanation. Several Climb operations are required along both background dimensions: (1) up to the Category level along the Stops dimension, to find stops corresponding to homes and airports; (2) up to theDay level along the Time dimension. Dice operations are finally used to filter out the sub-trajectories not occurring during the same day, and to keep only the trajectories going from a home to an airport. The transitive closure of the resulting sub-graph is finally computed.

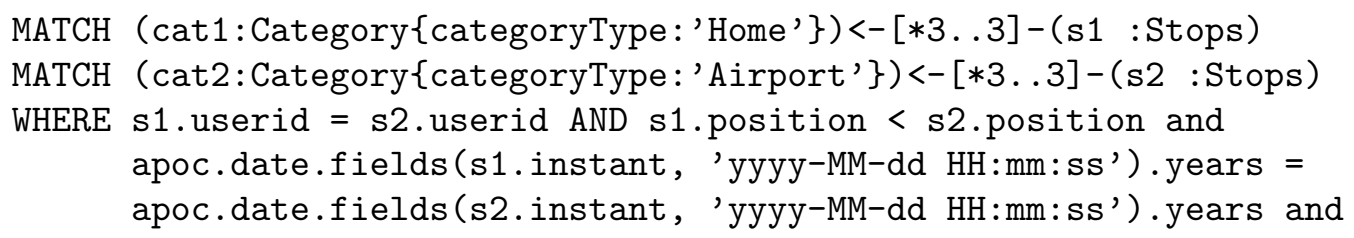




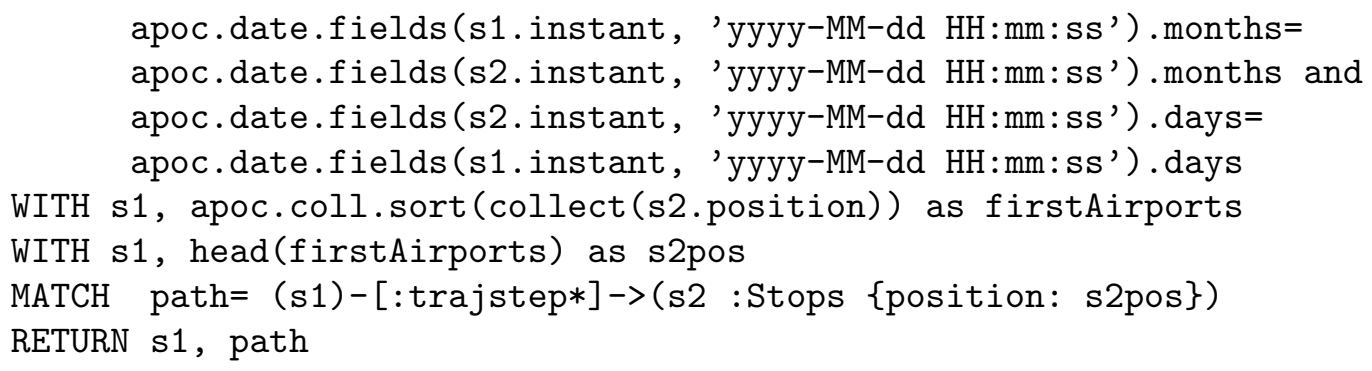

The climbings and dicings are computed in the first two parts of the query (the two MATCH clauses). Note that this is very intuitive, even for a the non-expert user. There are two tricky parts, however. First, the climbing along the Time dimension is performed through a conjunction of Boolean conditions over the instant property of the nodes of type Stop. The APOC library is used to operate with dates. The reason is that, in this case, this results more efficient than performing two matchings along the Time hierarchy as follows:

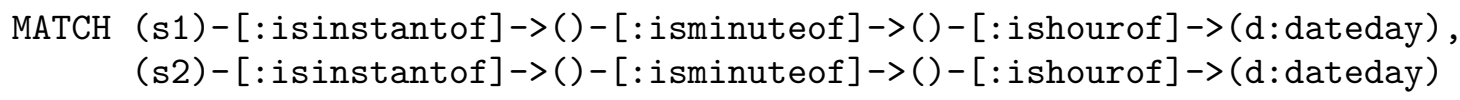

The second tricky part involved in this query is caused by the fact that the sequence of stops in the graph, at the Category aggregation level, can be of the form \{Home, Home, Airport, Airport, Airport...\}, and thus all combinations are included in the transitive closure. However, the query must only capture the first airport in that sequence. The "WITH s1, head(firstAirports)" clause does the job.

The next query partitions a trajectory into its sub-trajectories using the date as a partition function, by means of keeping only the longest subtrajectory occurring within a day. The Cypher expression is given in the appendix.

Query 11 For each day, and for each trajectory, find the longest sub-trajectory.

As a final example, the next query makes use of the trajectory graph, together with map information, to find out districts from where people travel to an airport, together with the part of the day in which this travel starts.

Query 12 Give the districts in which people leave from their home to the airport before 3pm.

The Cypher expression is also given in the appendix. 


\begin{tabular}{|c|c|c|}
\hline Type & Name & Size (\#) \\
\hline Node & Stops & 226,345 \\
\hline Node & Venues & 38,333 \\
\hline Node & Categories & 400 \\
\hline Node & Category & 33 \\
\hline Node & Dateminute & 128,412 \\
\hline Node & Datehour & 4,523 \\
\hline Node & Dateday & 252 \\
\hline Node & MonthYears & 11 \\
\hline Node & Years & 2 \\
\hline Edge & trajstep & 225,262 \\
\hline Egde & isVenue & 226,345 \\
\hline Edge & HasCategoryOf & 38,333 \\
\hline Edge & subCategoryOf & 400 \\
\hline Egde & isInstantOf & 226,345 \\
\hline Edge & isMinuteOf & 128,412 \\
\hline Edge & isHourOf & 4,523 \\
\hline Edge & isDayOf & 252 \\
\hline Edge & isMonthOf & 11 \\
\hline Total & \# Objects & $\mathbf{7 9 6 , 5 5 2}$ \\
\hline
\end{tabular}

Table 1: Number of nodes and edges in the graph database.

\subsection{Running the Queries on Neo4j and PostgresSQL}

Although query performance is not the core goal of this paper, the queries in Section 5.1 are run over the Neo4j database designed and populated as described in Section 4. Further, in order to compare performance against a relational alternative, the queries are written in the SQL language, and executed over a PostgresSQL database. The exception are the queries that involve spatial functions, which would be unfair to compare, since performance heavily depends on the spatial libraries used which, in the case of Neo4j are still in a first stage of development. Both databases are indexed in order to obtain the best possible query performance.

For the Neo4j database, the number of nodes and edges are given in Table 1. For the PostgresSQL database, there are two tables: Trajectories, with 226,252 tuples, and Categories, with 38,333 tuples.

Queries are run on a machine with a i7-6700 processor, 12 GB of RAM and a 250GB disk (actually, a virtual node in a cluster). The execution times reported are the averages of five runs of each experiment. 


\subsection{Discussion}

Although comparing performance of a graph database like Neo4j against relational databases with more than 20 years in the marketplace may seem, at first sight, unfair, it can give an idea of the potential of the former, even for the current state-of-the-art software. The discussion that follows is organised considering the query classes defined in Section 5.1. Results are summarized in Table 2. In the discussion, some of the queries will be expressed in SQL, although not all of them, since it is assumed that the reader has at least a basic knowledge of SQL.

For non-recursive pattern queries, writing the Cypher query implies just writing the pattern the user wants to check, in a very simple and intuitive way, provided the user has a basic knowledge. In addition to this, these kinds of queries are very efficient on a graph database, which can be observed in Table 2. Queries 1 and 2 are executed in Neo4j in 0.18 and 0.41 seconds, respectively, while the SQL equivalents take 0.3 and 05 seconds to execute. The reason is that the SQL queries require performing several joins, and reads, as shown below for Query 1, while joins are solved by direct path navigation in Neo4j.

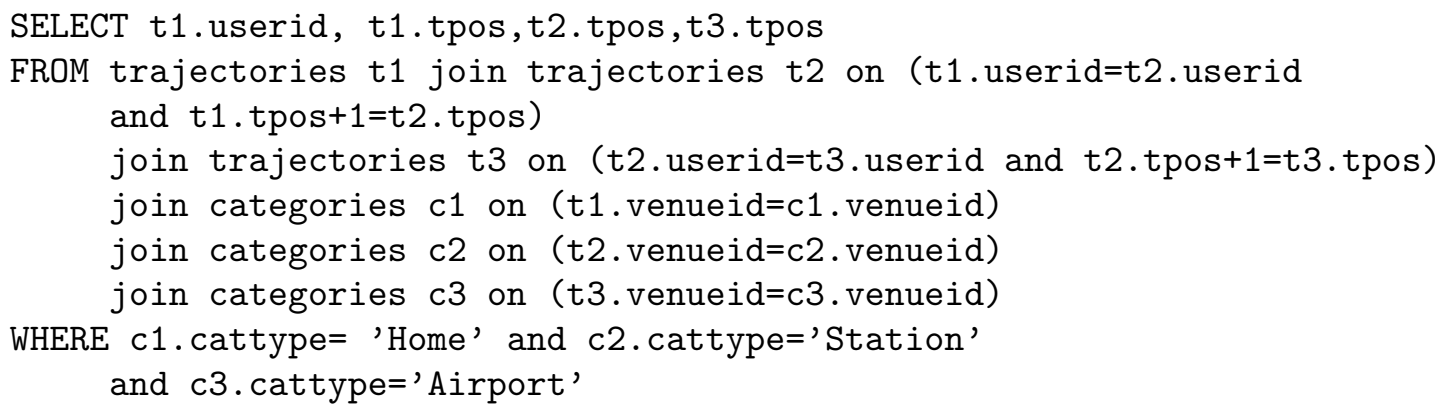

For queries computing travelled distance, Query 3 also performs better on the graph alternative than on the relational one. This Neo4j query directly looks for all pairs of stops, and then computes the distance between each pair. This is performed, on average, in 2.2 seconds, while just the join in PostgresSQL takes 31 seconds (as explained above, the geographic part has not been evaluated in the Postgres version). That is, when the SQL query requires joining and/or a sorting the complete database, performance tends to benefit the graph alternative. Query 4, which combines patterns and distance computation, is also a good example of the former. In this case, the pattern just consists in checking two kinds of stops (going from home to the airport, in this case). The navigation along the Stop dimension is performed very fast, as well as the distance computation. The query takes 
0.1 seconds to complete, outperforming SQL which took (without distance computation), 0.25 secs.

For spatial queries, only the Neo4j alternative is evaluated, as explained above. For the case of Neo4j, results are just given for completeness since, clearly, the spatial plugin provided for Neo4j has still to be developed. Spatial queries are included here to give an idea of the potential of enhancing graph queries of any kind (e.g., transitive closure queries, pattern queries, etc.), with spatial functions. Spatial queries that require going through all the stops take, naturally, long times to execute, given the high number of stops in the dataset. For example, Query 6 takes just 22 seconds to execute, since it only queries the starting position of each trajectory. On the other hand, Query 5, which needs to go through all the stops in all trajectories, takes over 700 seconds.

Aggregation queries also require going through the whole database. Thus, the same observations above are also valid in this case. Query 7 takes 0.8 seconds to complete on Neo4j, while the SQL alternative takes 1 second. Both queries return 359 records. The reason is that the pattern matching performing the join in Neo4j (to climb up to the Category level) is more efficient than the SQL join. This is somehow compensated by the fact that SQL performs no additional join to climb up to the Month level, while the Cypher query actually performs such climbing. When the size of the result increases, the advantage for Neo4j increases, as it can be seen in the result for the other aggregation queries.

At this point, the reader may argue that there would be better design alternatives for the relational database. This is true for some queries (like Query 3), while a different design would be worse for other ones. And the same may occur for the graph model. The chosen relational design is a generic one, with denormalized dimensions, which favours navigation along the hierarchies, preventing joining dimension levels (this is called a "Star Schema" design in data warehousing jargon [Kimball1996]).

For transitive closure queries, it is worth remarking that Cypher has not yet included many functions that would be needed to compute paths when the transitive closure of the graph is involved. This is why, as explained, extra statements are needed to solve some queries (like in Query 10 to filter out unnecessary sub-paths). Query 10 takes 7.8 seconds to execute on the graph database, while the SQL alternative (see the appendix), which requires recursion techniques, takes 1 minute. This suggests that for transitive closure queries, the graph database alternative can be competitive and in some cases even better than the recursive SQL solution. Of course, this is not conclusive, since many other factors impact on the results. For example, Query 10 asks for paths between home and airports. If this changes for more common stops like Restaurant and Banks, since there are more redun- 
dant paths to eliminate (due to the Cypher limitations commented above), performance decreases to 27 secs in Neo4j (this is denoted alternative 2 in Table 2), while the SQL query is not significantly affected. In both cases, however, Neo4j performs better than Postgres, and these results repeat for many different combinations of filters. This is due to the fact that the transitive closure is not computed over the complete graph. There is a situation, however, when the relational alternative is still better than the graph one. This is the case when transitive closure computation is performed over the whole graph, like in Query 11. When this computation is required, parallel graph computation is needed (Neo4j does not scale horizontally). Note that trajectory graphs are excellent candidates for parallelization, since the transitive closure of each trajectory can be computed independently of the others. On the other hand, it can be seen that the SQL query is very efficient for this task, and the query times are stable. These results suggest, then, that when the transitive closure is computed over a relatively small portion of the trajectory graph, the graph database alternative works better than the relational ones, but when it must be taken over the whole graph, the relational database performs better. However, for the same reasons, graph parallelization solutions are great candidates for this problem, particularly for trajectory graphs. A study of these alternative is beyond the scope of this paper.

Table 2 summarizes the test results. The last column on the right gives the ratio between the execution times on Neo4j and PostgresSQL. The best execution times for each query have been highlighted in bold font. As a conclusion, it can be said that for most kinds of queries, the graph database outperforms the relational one, the exception being the transitive closure queries requiring computing the closure of the whole trajectory graph. In this case, the relational alternative is clearly better. However, when the closure is taken over a small portion of the database, the results are competitive, or even favour the graph option.

\section{Open Problems and Future Work}

This paper discussed the problem of querying a collection of semantic trajectories modelled as a property graph and stored in a graph database, focusing on analytical queries, that imply aggregating the trajectories up to different granularity levels. Typically, trajectories are stored in a relational database. Given that trajectories can be seen as a graph, modelling and storing them as graphs instead of relations sound natural, and merits studying the plausibility of this solution. The outcome of this study suggests, from a qualitative point of view, that analytical trajectory queries are more 


\begin{tabular}{|c|c|c|c|c|}
\hline 1 & $2 \#$ & 3 & 4 & 5 \\
\hline Type of Query & Query $\neq$ & Neo4j (sec) & Postgres (sec) & $3 / 4$ \\
\hline Non-recursive pattern & 1 & 0.18 & 0.3 & 0.6 \\
\hline Non-recursive pattern & 2 & 0.41 & 0.5 & 0.82 \\
\hline Travelled distance & 3 & 2.2 & $31(\mathrm{w} / \mathrm{o}$ distance $)$ & 0.07 \\
\hline Distance \& pattern & 4 & 0.1 & 0.25 & 0.4 \\
\hline Spatial & 5 & 720 & $\mathrm{~N} / \mathrm{A}$ & $\mathrm{N} / \mathrm{A}$ \\
\hline Spatial & 6 & 22 & $\mathrm{~N} / \mathrm{A}$ & $\mathrm{N} / \mathrm{A}$ \\
\hline Aggregation & 7 & 0.8 & 1 & 0.8 \\
\hline Aggregation & 8 & 1.8 & 4 & 0.45 \\
\hline Aggregation \& distance & 9 & 2.4 & $\mathrm{~N} / \mathrm{A}$ & $\mathrm{N} / \mathrm{A}$ \\
\hline Transitive closure & 10 (alt. 1 & 7.8 & 60 & 0.13 \\
\hline Transitive closure & $\overline{10}$ (alt. 2 & 27 & 60 & 0.45 \\
\hline Transitive closure & 11 & 99 & 59 & 1.67 \\
\hline Spatial \& transitive closure & $\overline{12}$ & 970 & $\mathrm{~N} / \mathrm{A}$ & $\mathrm{N} / \mathrm{A}$ \\
\hline
\end{tabular}

Table 2: Execution times for the example queries.

naturally expressed over graphs, using a graph query language (in this case, Cypher, the query language for Neo4j), than over a relational representation. Moreover, from a quantitative point of view, for three out of the five classes of queries studied (non-recursive patterns, distance, spatial, aggregation, and transitive closure queries), the graph database queries run from 1.2 to 7 times faster than the relational ones. Only transitive closure queries that must go through the complete graph delivered better performance over the relational database. However, when queries require computing the transitive closure of only a relatively small portion of the database, the graph database results competitive, and even outperforms the relational database.

The results reported here, however promising, leave plenty of room for more research work. As a first indication of the road to follow, note that even for the queries where results are not positive (basically transitive closure queries), such results are very likely to change considering parallel execution. There are many parallel processing graph databases (e.g., GraphFrames ${ }^{8}$, Janusgraph ${ }^{9}$ that may take advantage of the characteristics of trajectory graphs in order to, for example, compute the transitive closure of the trajectory graph in parallel, and aggregate the results after this computation. This may speed-up computation by orders of magnitude. Therefore, the continuation of the work presented will explore the benefits of using those kinds of graph databases.

\footnotetext{
8 https://graphframes.github.io/

gttp://janusgraph.org/
} 


\section{References}

[Alvares et al.2007] Alvares, L.O., et al., 2007. A Model for Enriching Trajectories with Semantic Geographical Information. In: 15th ACM International Symposium on Geographic Information Systems (ACM-GIS 2007) ACM, 162-169.

[Angles2012] Angles, R., 2012. A Comparison of Current Graph Database Models. In: Proceedings of ICDE Workshops, Arlington, VA, USA, $171-177$.

[Angles2018] Angles, R., 2018. The Property Graph Database Model. In: Proceedings of the 12th Alberto Mendelzon International Workshop on Foundations of Data Management, Cali, Colombia, May 21-25, 2018.

[Angles and Gutierrez2008] Angles, R. and Gutierrez, C., 2008. Survey of graph database models. ACM Comput. Surv., 40 (1), 1:1-1:39.

[Chen et al.2009] Chen, C., et al., 2009. Graph OLAP: a multi-dimensional framework for graph data analysis. Knowl. Inf. Syst., 21 (1), 41-63.

[du Mouza and Rigaux2005] du Mouza, C. and Rigaux, P., 2005. Mobility Patterns. GeoInformatica, 9 (4), 297-319.

[Giannotti et al.2007] Giannotti, F., et al., 2007. Trajectory Pattern Mining. In: Proceedings of Knowledge Discovery and Data Mining (KDD 2007) ACM, 667-680.

[Giannotti et al.2006] Giannotti, F., Nanni, M., and Pedreschi, D., 2006. Efficient Mining of Temporally Annotated Sequences. In: Proceedings of the Sixth SIAM International Conference on Data Mining (SDM 2006) SIAM.

[Gómez et al.2017] Gómez, L.I., Kuijpers, B., and Vaisman, A.A., 2017. Performing OLAP over Graph Data: Query Language, Implementation, and a Case Study. In: Proceedings of the International Workshop on Real-Time Business Intelligence and Analytics, BIRTE, Munich, Germany, August 28, 2017, 6:1-6:8.

[Gómez and Vaisman2013] Gómez, L.I. and Vaisman, A.A., 2013. Mining semantic trajectories. Intell. Data Anal., 17 (5), 857-898.

[Gryllakis et al.2017] Gryllakis, F., et al., 2017. Searching for SpatioTemporal-Keyword Patterns in Semantic Trajectories. In: Advances in Intelligent Data Analysis XVI - 16th International Symposium, IDA 2017, London, UK, October 26-28, 2017, Proceedings, 112-124. 
[Gryllakis et al.2018] Gryllakis, F., et al., 2018. Spatio-Temporal-Keyword Pattern Queries over Semantic Trajectories with Hermes@Neo4j. In: Proceedings of the 21th International Conference on Extending Database Technology, EDBT 2018, Vienna, Austria, March 26-29, 2018., 678-681.

[Güting and Schneider2005] Güting, R.H. and Schneider, M., 2005. Moving Objects Databases. Morgan Kaufman.

[Hartig2014] Hartig, O., 2014. Reconciliation of RDF* and Property Graphs. CoRR, abs/1409.3288.

[Karli and Saygin2009] Karli, S. and Saygin, Y., 2009. Mining Periodic Patterns in Spatio-temporal Sequences at Different Time Granularities. Intelligent Data Analysis, 13 (2), 301-335.

[Kimball1996] Kimball, R., 1996. The Data Warehouse Toolkit. J. Wiley and Sons.

[Leonardi et al.2014] Leonardi, L., et al., 2014. A general framework for trajectory data warehousing and visual OLAP. GeoInformatica, 18 (2), 273-312.

[Parent et al.2013] Parent, C., et al., 2013. Semantic trajectories modeling and analysis. ACM Comput. Surv., 45 (4), 42:1-42:32.

[Pelekis et al.2015] Pelekis, N., Sideridis, S., and Theodoridis, Y., 2015. Hermessem: A semantic-aware framework for the management and analysis of our LifeSteps. In: 2015 IEEE International Conference on Data Science and Advanced Analytics, DSAA 2015, Campus des Cordeliers, Paris, France, October 19-21, 2015, 1-10.

[Renso et al.2013] Renso, C., Spaccapietra, S., and Zimányi, E., eds. , 2013. Mobility Data: Modeling, Management, and Understanding. Cambridge University Press.

[Robinson et al.2013] Robinson, I., Webber, J., and Eifrém, E., 2013. Graph Databases. O'Reilly Media.

[Spaccapietra et al.2008] Spaccapietra, S., et al., 2008. A conceptual view on trajectories. Data Knowl. Eng., 65 (1), 126-146.

[Spaccapietra et al.2013] Spaccapietra, S., Parent, C., and Spinsanti, L., 2013. Trajectories and Their Representations. Mobility Data: Modeling, Management, and Understanding., 3-22. 
[Vaisman and Zimányi2014] Vaisman, A. and Zimányi, E., 2014. Data Warehouse Systems: Design and Implementation. Springer.

[Vaisman and Zimányi2013] Vaisman, A.A. and Zimányi, E., 2013. Trajectory Data Warehouses. Mobility Data: Modeling, Management, and Understanding., 62-82.

[Zhao et al.2011] Zhao, P., et al., 2011. Graph cube: on warehousing and OLAP multidimensional networks. In: Proceedings of the 2011 ACM SIGMOD International Conference on Management of Data, Athens, Greece ACM, 853-864.

\section{Appendix: Cypher and SQL Expressions for Queries in Section 5.1}

This appendix includes the Cypher or SQL expressions not included in the main body of the paper.

Query 5.2 Find the trajectories that go from a bar (or similar) to a restaurant, again to a bar (or similar) and end at a restaurant, without intermediate stops.

This query aims at comparing performance for long patterns.

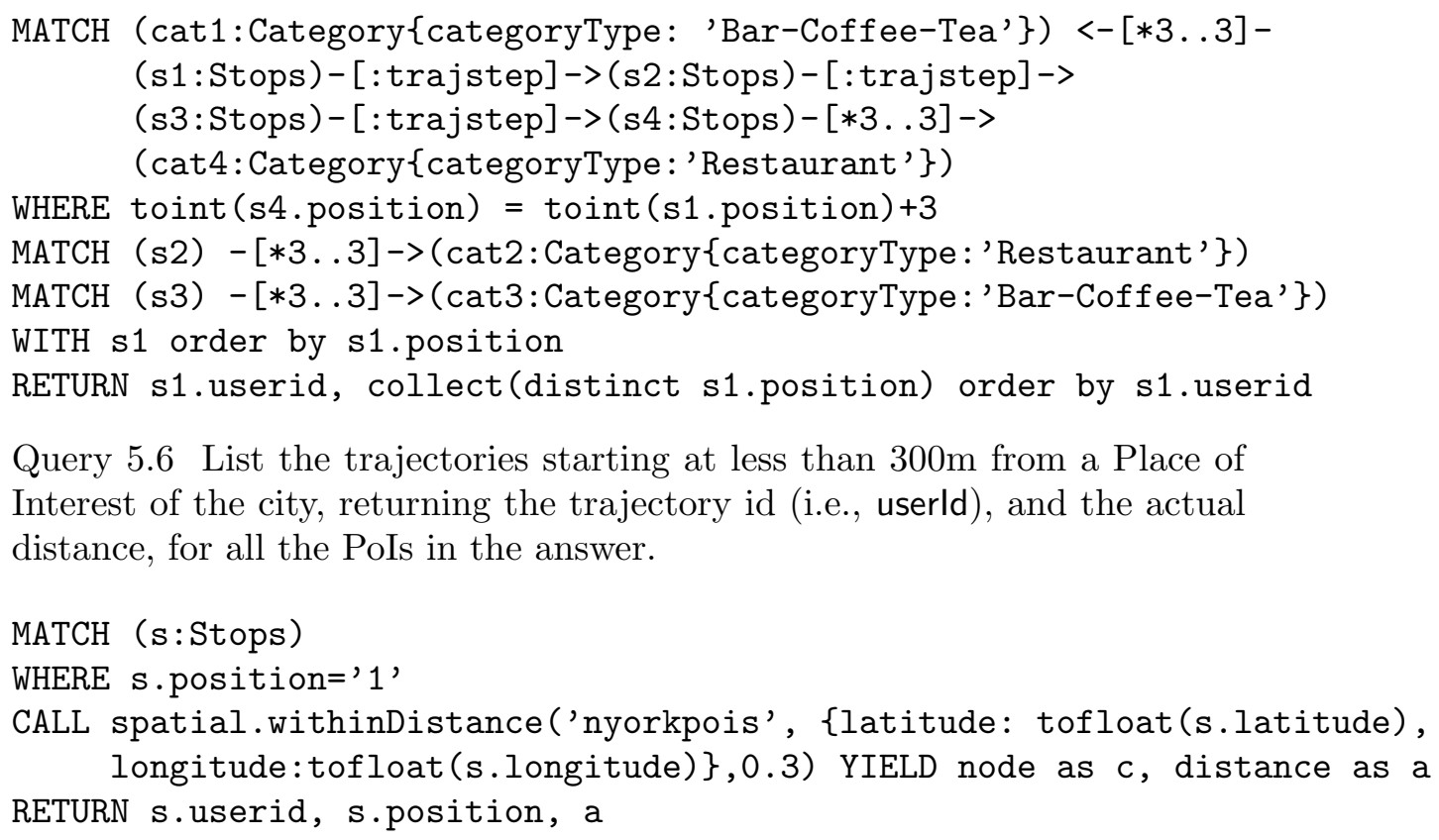

Query 5.6 List the trajectories starting at less than $300 \mathrm{~m}$ from a Place of Interest of the city, returning the trajectory id (i.e., userld), and the actual distance, for all the PoIs in the answer. 
Query 5.8 Compute the number of stops per day per user, along with the starting position of each sub-trajectory for each day.

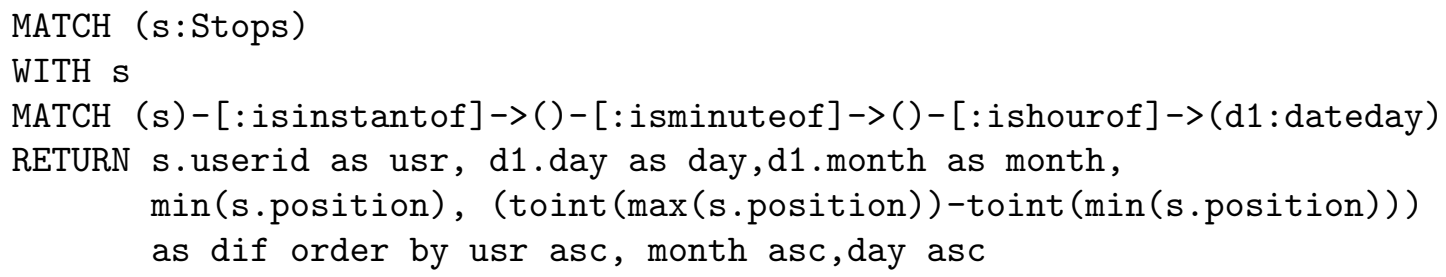

Query 5.9 For each trajectory, compute its total length, as the sum of the distances between each pair of stops.

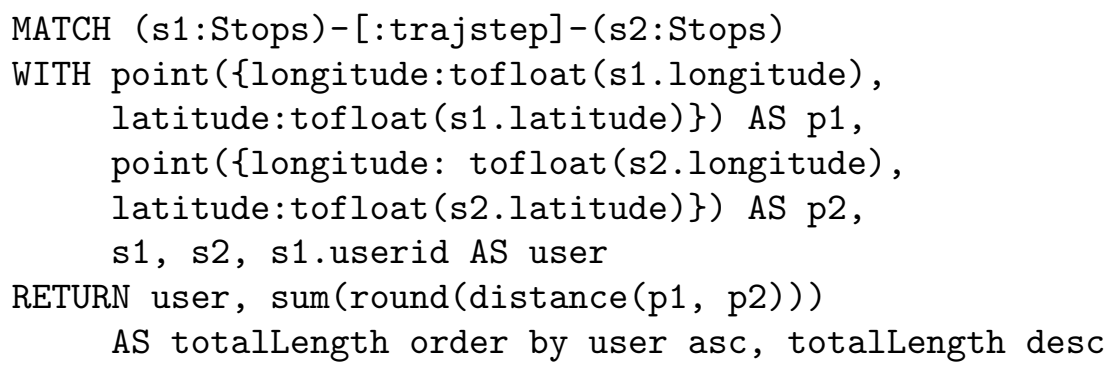

Query 5.10 For each trajectory, find the paths that go from a private home to an airport in the same day.

The Cypher expression is given in the main body of the paper. The SQL expression for this query reads as follows.

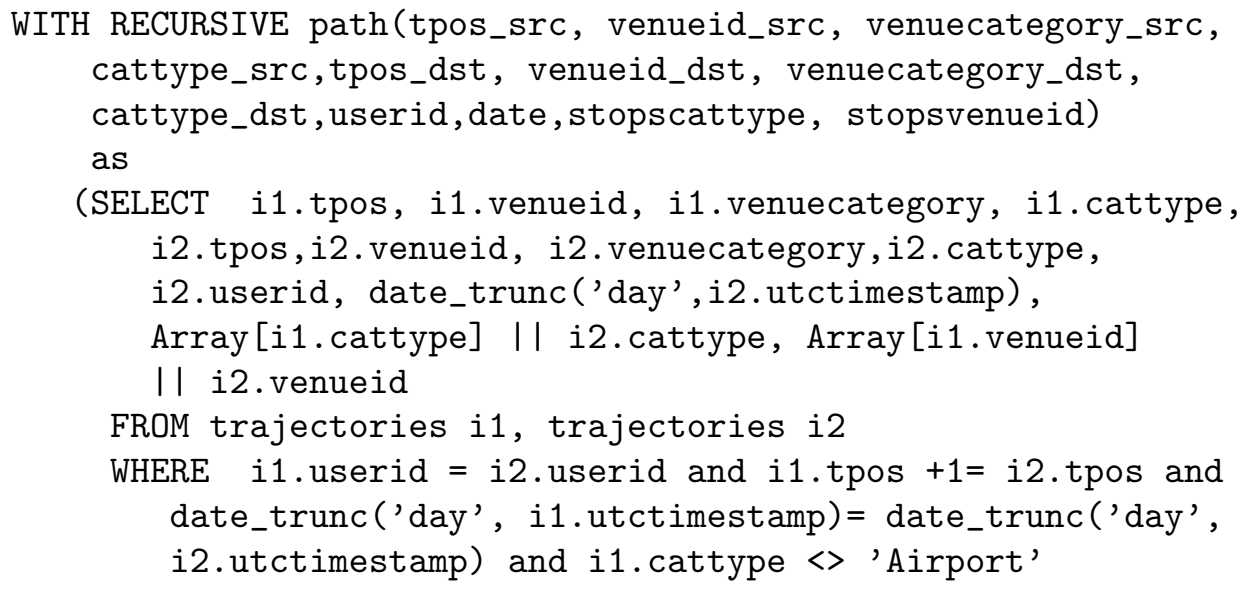


UNION ALL

SELECT path.tpos_src, path.venueid_src, path.venuecategory_src, path.cattype_src, i2.tpos, i2.venueid, i2.venuecategory, i2.cattype, i2.userid, date_trunc('day', i2.utctimestamp), stopscattype II Array[i2.cattype], stopsvenueid || Array [i2. venueid]

FROM path, trajectories i2

WHERE path.userid $=i 2$.userid and path.tpos_dst $+1=i 2 . t p o s$ and date_trunc ('day', path.date)=date_trunc ('day', i2.utctimestamp) and 'Airport' <> path.cattype_dst )

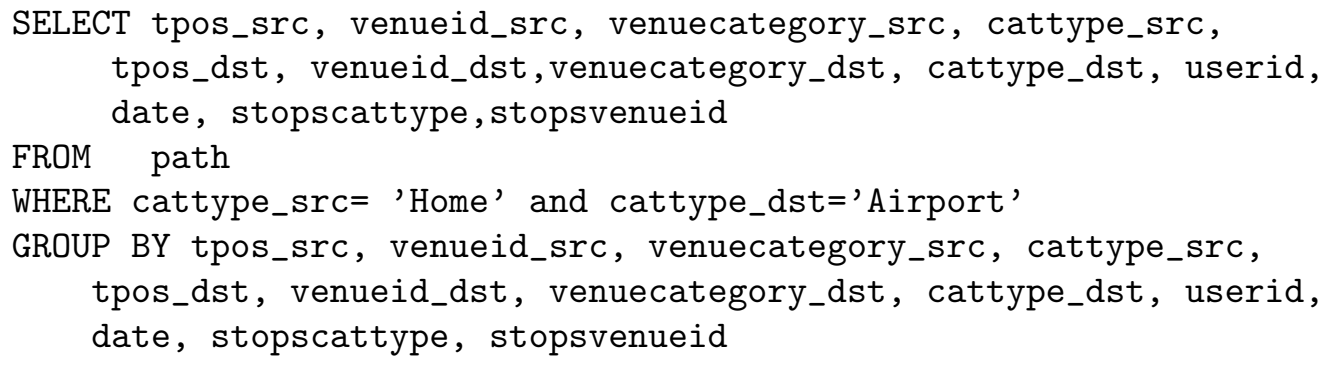

Query 5.11 For each day, and for each trajectory, find the longest subtrajectory.

Below, the corresponding Cypher query is shown.

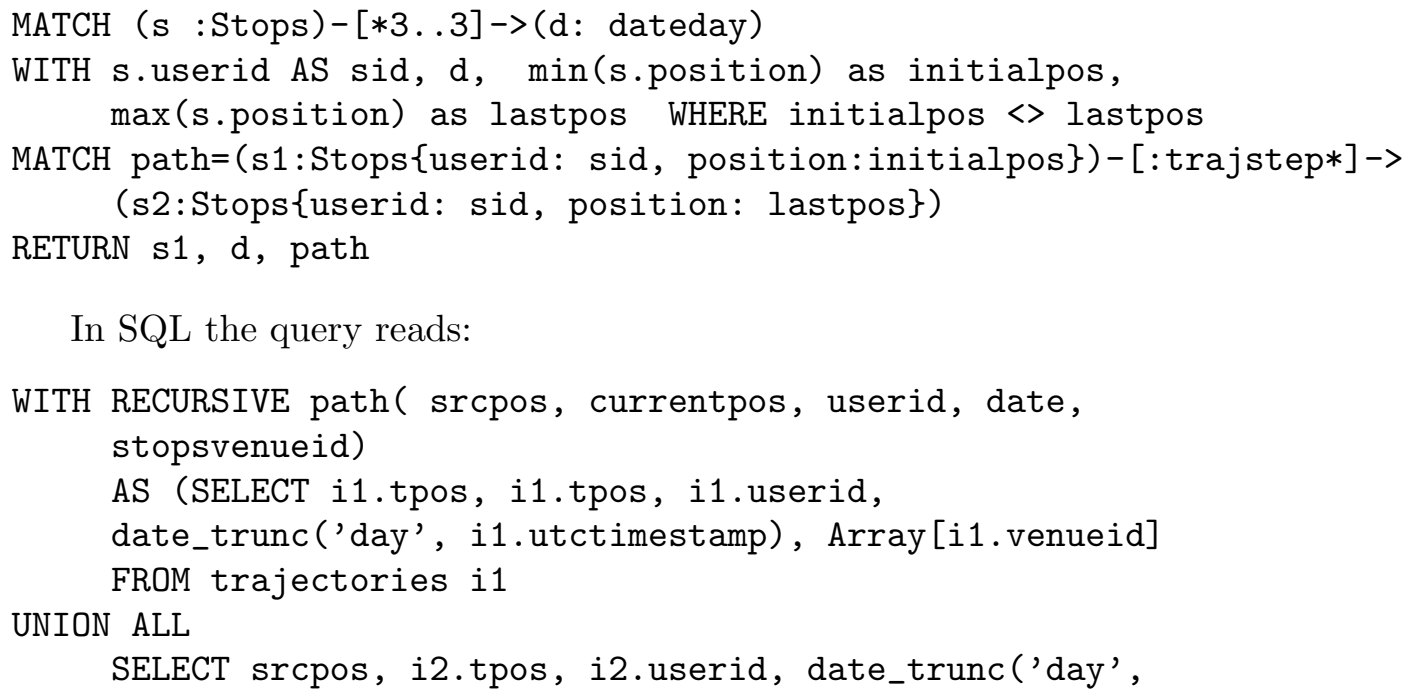

In SQL the query reads:

WITH RECURSIVE path( srcpos, currentpos, userid, date, stopsvenueid)

AS (SELECT i1.tpos, i1.tpos, i1.userid, date_trunc('day', i1.utctimestamp), Array[i1.venueid] FROM trajectories i1

UNION ALL

SELECT srcpos, i2.tpos, i2.userid, date_trunc('day', 


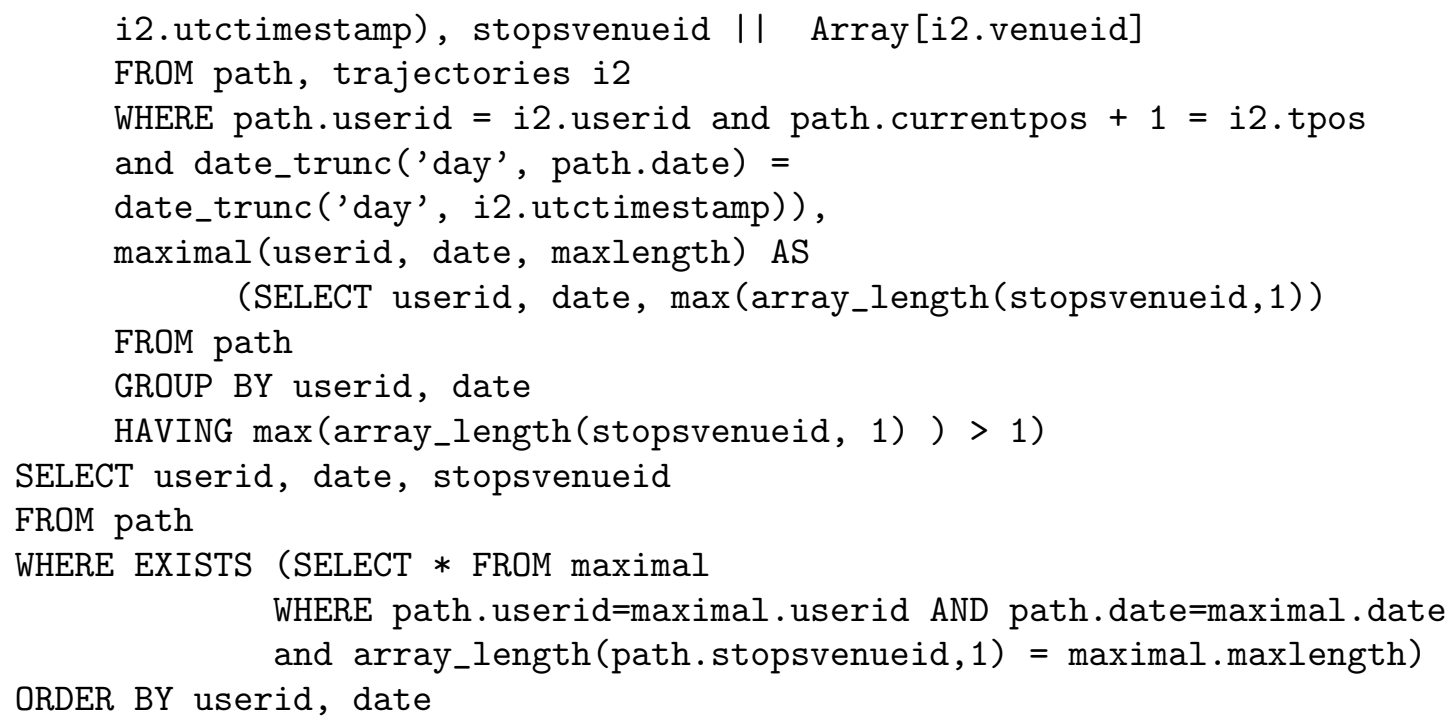

Query 5.12 Give the districts in which people leave from their home to the airport before $3 \mathrm{pm}$.

The query makes use of additional layers, in this case, the layer containing the districts. The query in Cypher is expressed as follows .

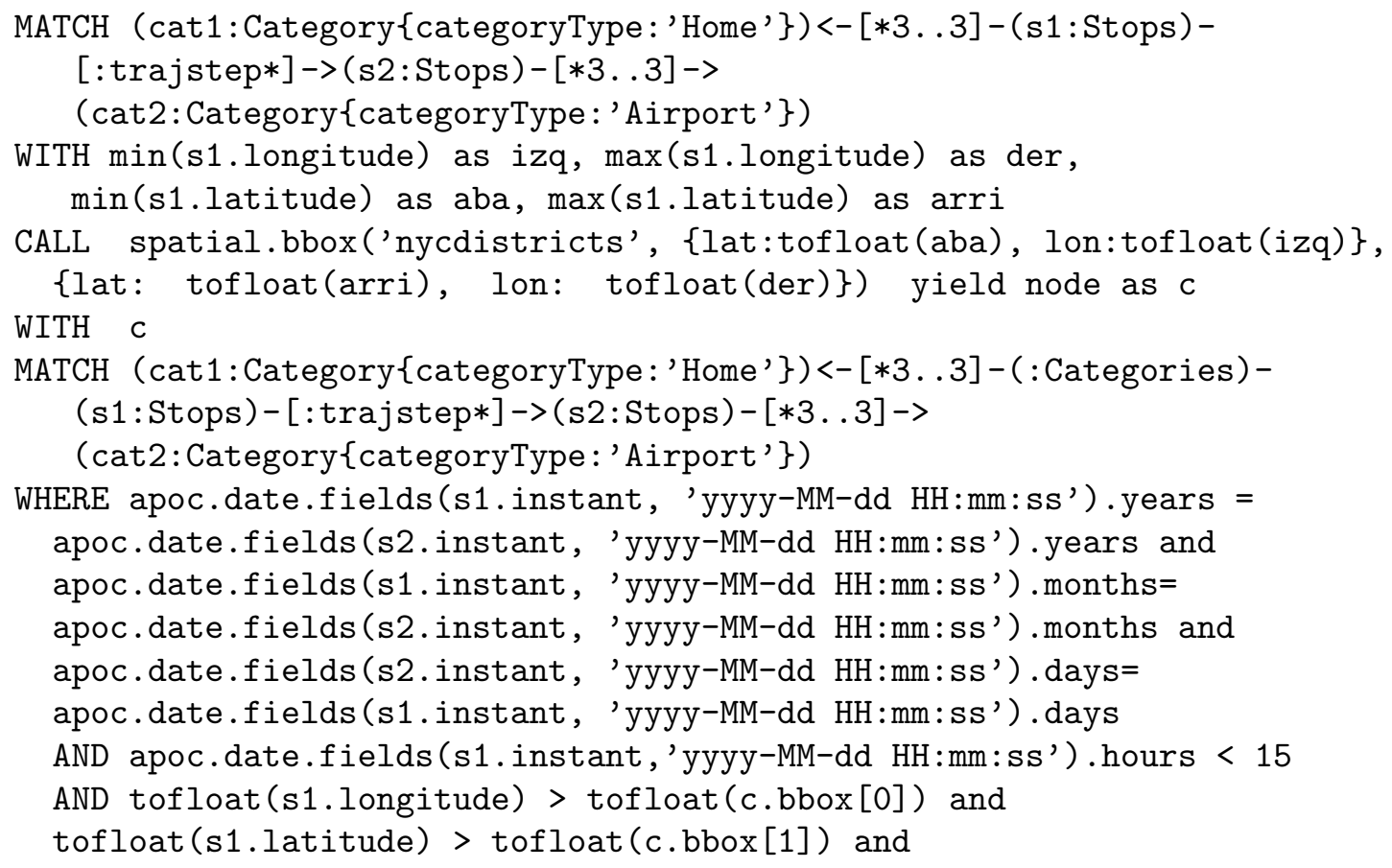


tofloat (s1.longitude) < tofloat (c.bbox [2])

and tofloat(s1.latitude) < tofloat (c.bbox[3])

RETURN s1, s2, c.NAME

The Neo4j spatial plugin still does not provide a function that can compute if a point is contained inside a polygon. Therefore, this query is solved using the bounding boxes of the districts, as can be seen in the last part of the query. The first part computes the bounding box containing all stops in the dataset. Then, the spatial.bbox function returns all the district in this bounding box. Then, all trajectories leaving from a home to an airport before $3 \mathrm{pm}$ are computed. Finally, the districts corresponding at these homes are computed (based on the bounding box of the district geometry). 